\title{
Éléments d'analyse du marquage différentiel de l'objet dans les langues romanes
}

\begin{abstract}
Alexandru Mardale*
\section{INTRODUCTION}

C'est un fait bien connu que dans certaines langues romanes les objets directs ayant certaines propriétés spécifiques peuvent être marqués par une préposition, d'où le nom de ce phénomène : le marquage différentiel de l'objet (dorénavant MDO $)^{1}$.

Dans cet article, nous nous proposons de décrire ce phénomène dans les trois langues romanes qui en font un usage systématique : le roumain (qui emploie la préposition $p e$ ), l'espagnol et le sarde (qui emploient la préposition $a$ ). Notre but est de mettre en évidence les similitudes et les différences que revêt le phénomène dans ces trois langues. Par ailleurs, nous discuterons de façon plus approfondie une généralisation qui rende compte de son existence. Cette généralisation a déjà été esquissée par certains auteurs (voir Laca (1995), Dobrovie-Sorin $(1997,2002)$, Bleam $(2004,2005)$, Cornilescu \& DobrovieSorin (2007)) et elle a pour base la corrélation entre le marquage de l'objet et le type de dénotation de ce dernier.

Cet article est organisé comme suit. Dans la section 2, nous introduirons les données à analyser. Nous commencerons par examiner brièvement les paramètres qui déterminent le MDO, en adoptant la distinction de Laca (2002, 2006), entre paramètres locaux et paramètres globaux. Ainsi, nous examinerons ce phénomène en fonction des paramètres locaux (sections 2.1.1 à 2.1.3). Ensuite, nous nous intéresserons à quelques situations de marquage spécifique

\footnotetext{
*alexandru.mardale@inalco.fr

${ }^{1}$ Nous avons emprunté ce terme à Bossong (1985). En effet, ce terme est un terme plus général, qui désigne des constructions similaires dans des langues variées (voir, par exemple, Lazard (1982), Karimi (1990, 1996), Shokouhi \& Kipka (2003) pour le persan ; de Hoop \& Narashimhan (2005) pour le hindi; Rapoport (1995) pour l'hébreu; Nikolaeva (2001) pour l'ostyak; Enç (1991), Erteschik-Shir (1997), von Heusinger \& Kornflit (2005) pour le turc; Escandell-Vidal (2007) pour le catalan baléaire ; Lois (1982), Iemmolo (2007) pour certains dialectes de l'italien). Pour parler de ce même phénomène dans les langues romanes, d'autres auteurs parlent d'accusatif prépositionnel ou encore d'objet direct prépositionnel (voir, entre autres, Niculescu $(1959,1965)$ ).
} 
déterminé par des paramètres globaux (sections 2.2.1 à 2.2.4). Après la description des données, nous ferons le point sur les résultats obtenus, que nous interpréterons comme donnant lieu à deux types de variation : une variation forte (section 3.1) et une variation faible (section 3.2). La section 4 passera en revue les analyses précédentes. La section 5 proposera une solution alternative pour l'analyse du MDO dans les langues romanes, à savoir une généralisation corrélant l'apparition de la marque au type dénotationnel de l'objet direct. Finalement, dans la section 6 , nous résumerons les conclusions de notre étude.

\section{LES DONNEES ET LES PARAMETRES}

Voyons d'abord quelques exemples qui illustrent brièvement le $\mathrm{MDO}^{2}$. Rappelons qu'en sarde, tout comme en espagnol, la marque introduisant l'objet direct est morphologiquement identique à la préposition $a$ « à » :

$$
\begin{aligned}
& \text { L-am întâlnit *(pe) Ion. } \\
& \text { le } \mathrm{e}_{\mathrm{CL} . \mathrm{Acc}} \text {-ai rencontré } \mathrm{PE}_{\mathrm{Acc}} \text { Jean }
\end{aligned}
$$

(roumain)

$$
\begin{aligned}
& \text { Vi *(a) Juan } \\
& \text { vis } \mathrm{A}_{\text {Acc }} \text { Jean } \\
& \text { « J'ai vu Jean. » }
\end{aligned}
$$

(espagnol)

(sarde)

Dans ces exemples, le MDO est obligatoire, mais dans d'autres cas il peut aussi être optionnel ou exclu, en fonction de différents paramètres.

Les paramètres qui déterminent le MDO en général, et l'accusatif prépositionnel dans les langues romanes en particulier, peuvent être organisés dans une hiérarchie comportant les trois paramètres suivants (cf. Comrie (1975), Bossong (1985), Croft (1990), Lazard (1994), Laca (2002, 2006), Aissen (2003), von Heusinger \& Kaiser (2005)) :

(i) le caractère animé de l'objet direct,

(ii) le caractère spécifique de l'objet direct,

(iii) la topicalisation de l'objet direct.

À chacun de ces paramètres correspondent plusieurs valeurs graduelles que nous examinerons brièvement dans ce qui suit.

Avant de procéder à cet examen, nous voudrions rappeler une observation de Laca $(2002,2006)$ dont nous tiendrons compte dans la présentation des données.

\footnotetext{
2 Notons que dans les exemples comportant la convention *(a/pe), le MDO est obligatoire ; dans ceux qui comportent la convention (a/pe), le MDO est facultatif, tandis que dans ceux qui comportent la convention $(* a / p e)$, le MDO est exclu.
} 
L'auteur regroupe les deux premiers paramètres dans une classe qu'elle appelle «paramètres locaux ». Ce sont les paramètres qui concernent les propriétés inhérentes du nom objet direct (voir la section 2.1 ci-dessous). Le troisième paramètre doit être compris dans un sens plus large. Il concerne les facteurs externes à l'objet, plus précisément les propriétés du contexte dans lequel l'objet apparaît (voir la section 2.2 ci-dessous). C'est pour cette raison que ces facteurs ont été regroupés dans une classe appelée des «paramètres globaux ».

\subsection{Les paramètres locaux}

Tout d'abord, rappelons que les paramètres locaux se réfèrent aux propriétés inhérentes de l'objet direct (c.-à-d. les propriétés grammaticales et lexicales du nom objet direct). Afin de les examiner, nous adopterons les résultats des analyses d'Aissen (2003) et Laca $(2002,2006)$. Ces auteurs proposent de prendre en considération non seulement les valeurs qui tiennent au caractère animé ou spécifique $^{3}$ du référent de l'objet, mais aussi la catégorie grammaticale de ce dernier. Plus précisément, Laca (2006) propose de croiser les différentes valeurs pour obtenir les échelles suivantes :

(A) pronoms [+ humain $]>$ noms propres [+ humain $]>$ pronoms $[+$ animé $]$

(B) GN définis [+ humain] $>$ noms propres [+ animé] $>$ pronoms [- animé]

(C) GN indéfinis spécifiques [+ humain] $>$ GN définis [+ animé $]>$ noms propres [- animé]

(D) GN non spécifiques [+ humain] $>\mathrm{GN}$ indéfinis spécifiques [+ animé] $>$ GN définis [- animé]

(E) GN non spécifiques [+ animé] $>$ GN indéfinis spécifiques [- animé] $>$ GN non spécifiques [- animé]

Sur la base de ces échelles, on peut faire des prédictions quant à la possibilité ou l'impossibilité d'apparition de la marque.

\footnotetext{
${ }^{3}$ Depuis les études de Farkas $(1995,2002)$, il est couramment admis qu'il existe différents types de spécificité : (i) spécificité partitive, (ii) spécificité de portée, (iii) spécificité épistémique. Nous ne rentrerons pas dans le détail des définitions de ces termes. Cependant, nous voudrions rappeler une observation due à Leonetti (2003), selon laquelle aucun de ces types particuliers n'est systématiquement corrélé au marquage différentiel de l'objet. Pour analyser ce phénomène, il est souhaitable d'adopter une définition plus large, d'ordre pragmatique, pour deux raisons, au moins. La première consiste en le fait que les types de spécificité en question sont le résultat contextuel de différents processus sémantiques ou syntaxiques. La seconde est que la marque de l'objet direct n'est pas exclusive de l'un ou de l'autre type. Par conséquent, nous utiliserons le terme "spécifique» dans une acception pragmatique, plus précisément pour désigner l'intention du locuteur d'utiliser une certaine expression afin de renvoyer à un référent particulier. Notons que cette acception n'exclut pas les autres définitions du terme et qu'elle se rapproche notamment de la définition de la spécificité «épistémique » chez Farkas (op. cit.). Par ailleurs, aux notions évoquées on pourrait ajouter une notion similaire, celle d'autonomie référentielle (Danon-Boileau \& Donabédian (1993)).
} 
En règle générale, on admet qu'un objet qui occupe une position supérieure dans la hiérarchie (voir les positions qui se trouvent à gauche des échelles A-C) a de fortes chances d'être marqué. À l'inverse, un objet qui occupe une position inférieure dans la hiérarchie (voir les positions qui se trouvent à droite des échelles C-E) a de faibles chances d'être marqué (cf. aussi von Heusinger \& Kaiser (2005 : 38)).

Autrement dit, le MDO va du haut vers le bas des échelles citées et de la gauche vers la droite de ces mêmes échelles.

Dans ce qui suit, nous examinerons de façon détaillée les données des langues mentionnées à l'aide des échelles A-E que nous ajusterons en fonction des besoins de notre présentation. Notre but est de mettre en évidence les similitudes et les différences entre les trois langues quant aux propriétés inhérentes de l'objet direct.

Comme nous l'avons déjà suggéré, ces langues ont ceci en commun qu'elles sont toutes sensibles aux paramètres locaux, en ce sens qu'elles marquent les objets qui occupent une position supérieure dans la hiérarchie (c.-à-d. les pronoms [+ humain] et les noms propres [+ humain]). De même, elles ont en commun de ne pas marquer les objets qui occupent une position inférieure dans la hiérarchie (c.-à-d. les groupes nominaux indéfinis spécifiques [- animé] et les groupes nominaux non spécifiques [- animé]). D'autre part, elles présentent des différences en ce qui concerne le marquage des objets occupant les positions du milieu de la hiérarchie (cf. les échelles B, C et D).

\subsubsection{Le roumain}

La plupart des exemples de cette sous-section viennent de notre propre usage. Certains d'entre eux sont adaptés de Niculescu (1965), GLR (1966), DobrovieSorin (1987a, 1994), Cornilescu (2000), Farkas \& von Heusinger (2003), GALR (2005), von Heusinger \& Onea (2007). Nous précisons qu'il s'agit ici d'énoncés déclaratifs sans contrainte spécifique de contexte (contrairement aux exemples qui seront évoqués dans la section sur les facteurs globaux).

\section{$P E$ est obligatoire avec les objets directs réalisés comme :}

I. Pronoms : personnels toniques [+ humain] (4), déictiques et anaphoriques [+/- animé] (5)

$$
\begin{aligned}
& M_{i} \text {-a invitat *(pe) } \text { mine }_{i}
\end{aligned}
$$

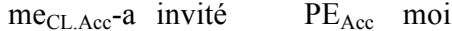

$$
\begin{aligned}
& \text { «Il / elle m'a invité. » }
\end{aligned}
$$

(5) a. $L_{i}$-am văzut *(pe) acesta $_{i} . \quad$ (acesta = l'étudiant, le cheval, le film) le $_{\text {CL.Acc }}$-ai vu $\quad \mathrm{PE}_{\mathrm{Acc}}$ celui-ci « J'ai vu celui-ci. »

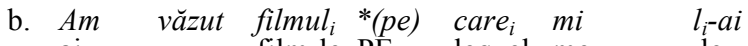

ai vu film-le PE $\mathrm{PE}_{\mathrm{Acc}}$ lequel $\mathrm{me}_{\mathrm{CL} . \mathrm{Dat}} \mathrm{le}_{\mathrm{CL} . \mathrm{Acc}}$-as




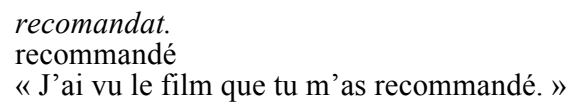

II. Noms propres [+ animé] (6) et GN définis assimilables aux noms propres [+ humain] (7)

$$
\begin{aligned}
& \begin{array}{llll}
L_{i} \text {-am } & \text { chemat } & *(p e) & \text { Lupu }_{i} \text {. } \\
\text { le }_{\text {CL.Acc-ai }} & \text { appelé } & \mathrm{PE}_{\mathrm{Acc}} & \text { Lupu }
\end{array} \\
& \text { « J'ai appelé Lupu. » }
\end{aligned}
$$

(7) Am văzut-o *(pe) mama $_{i}$.

ai vu-la $a_{C L}$.Acc $P E_{\text {Acc }}$ mère-la

" J'ai vu ma mère. »

\section{Les quantifieurs nus [+ humain]}

(8) N-am văzut *(pe) nimeni.

ne-ai vu PE $\mathrm{PE}_{\text {Acc }}$ personne

« Je n'ai vu personne. »

\section{$P E$ est optionnel avec :}

\section{Les GN définis spécifiques [+ humain]}

(9) $\quad \begin{array}{llll}\text { Am } & \text { căutat }\left(-o_{i}\right) & \text { (pe) } & \text { studentă } \breve{a}_{i}{ }^{4} \\ \text { cherché-la }\end{array}$

"J'ai cherché l'étudiante. »

\section{$P E$ est exclu avec:}

I. Les GN spécifiques : noms propres [- animé] (10), GN définis [- humain] (11)

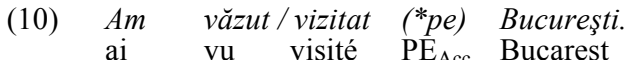

« J'ai vu / visité Bucarest. »

\footnotetext{
${ }^{4}$ Rappelons que les groupes nominaux du type donné en (9) ont une interprétation définie malgré la chute de l'article défini (voir Mardale $(2006,2007)$ pour l'analyse de ce phénomène en roumain). L'article défini est visible si l'on accompagne le groupe nominal en question d'un modifieur (e.g. Am căutat $\left(-o_{i}\right)$ (pe) studenta $a_{i}$ din prima bancă. ai cherché-la $\mathrm{CL}_{\mathrm{C} . \mathrm{Acc}} \mathrm{PE}_{\mathrm{Acc}}$ étudiante-la de-en première-la pupitre «J'ai cherché l'étudiante du premier rang »). Nous ne donnons pas ici des noms avec modifieurs pour éviter de créer des confusions avec les données qui seront examinées dans la section concernant les facteurs globaux (voir, plus précisément, la sous-section 2.2.4). Cette même observation vaut également pour les exemples roumains (11) et (15) ci-dessous, de même que pour les exemples correspondants en espagnol et en sarde.
} 
(11) Am chemat $\left({ }^{*} p e\right)$ pisică. ai appelé $\mathrm{PE}_{\mathrm{Acc}}$ chatte

« J'ai appelé le chat. »

II. Les GN non spécifiques [+/- animé] : GN indéfinis ${ }^{5}$ (12), noms nus (13)

(12) a. Caut (*pe) un student. cherche $\mathrm{PE}_{\mathrm{Acc}}$ un étudiant " Je cherche un étudiant. »

b. Caut (*pe) un elefant / o carte. cherche $\mathrm{PE}_{\mathrm{Acc}}$ un éléphant unelivre « Je cherche un éléphant / un livre. »

(13) a. Angajez (*pe) secretară. embauche $\mathrm{PE}_{\mathrm{Acc}}$ secrétaire « J'embauche (une) secrétaire. »

b. Caut (*pe) reviste. cherche $\mathrm{PE}_{\mathrm{Acc}}$ magazines « Je cherche des magazines. »

\title{
III. Les quantifieurs nus [- humain]
}

(14) $\quad N$-am văzut (*pe) nimic. ne-ai vu $\mathrm{PE}_{\mathrm{Acc}}$ rien « Je n'ai rien vu. »

\section{Les GN génériques [+/- animé]}

\author{
$\begin{array}{llll}\text { (15) a. Ion } & \text { adoră } / \text { respectă } & \left({ }^{*} p e\right) & \text { fete. } \\ \text { Jean } & \text { adore } / \text { respecte } & \mathrm{PE}_{\mathrm{Acc}} & \text { filles }\end{array}$ \\ "Jean adore / respecte les filles. " \\ b. Ion adoră (*pe) câini. \\ Jean adore $\mathrm{PE}_{\mathrm{Acc}}$ chiens \\ « Jean adore les chiens.»
}

\begin{abstract}
${ }^{5}$ Dans cette section, nous considérons que les groupes nominaux indéfinis, quand ils ne comportent pas de modifieur(s), ont une lecture prototypique non spécifique. La lecture spécifique est bien évidemment possible et elle est plus particulièrement visible quand les indéfinis sont accompagnés d'un modifieur (e.g., Caut (pe) un student care stie engleză cherche $\mathrm{PE}_{\mathrm{Acc}}$ un étudiant qui sait anglais « Je cherche un étudiant qui parle l'anglais). Ce genre de construction sera examiné dans la section concernant les paramètres globaux (voir $§ 2.2$ ci-dessous).
\end{abstract}




\subsubsection{L'espagnol ${ }^{6}$}

La plupart des données de cette section nous ont été fournies par des locuteurs natifs parlant l'espagnol standard d'Espagne'. Certaines d'entre elles sont adaptées de Roegiest (1979), Lois (1982), Laca (1995, 2002, 2006, c.p.), Pensado (1995), Torrego Salcedo (1999), Leonetti (2003, 2007, c.p.), von Heusinger \& Kaiser $(2005,2007)$.

$A$ est obligatoire avec les objets directs réalisés comme:

(16) $\operatorname{Lo}_{i} \quad$ arrestaron $*(a) \quad e ́ l_{i}$ le $_{\text {CL.Acc }}$ arrêtèrent $\mathrm{A}_{\mathrm{Acc}}$ lui « Ils l'ont arrêté. »

(17) Escucho *(a)l mío. (el mio = mon enfant, mon collègue $)$ entends $\mathrm{A}_{\mathrm{Acc}}-\mathrm{le}$ mien « J'entends / écoute le mien. »

II. GN spécifiques : noms propres [+ animé] (18), GN définis [+ humain] (19)

(18) Juan mató *(a) Dorotea. Jean tua $A_{\text {Acc }}$ Dorothée « Jean a tué Dorothée. »

(19) a. No hemos visto *(a) su padre. ne avons vu $A_{A c c}$ son père. « Nous n'avons pas vu son père. »

b. Juan insulta *(a)l señor. Jean insulte $\mathrm{A}_{\mathrm{Acc}}-\mathrm{le} \quad$ Monsieur « Jean insulte le Monsieur. »

\section{Les quantifieurs nus [+ humain]}

Escucho *(a) alguien. entends $\mathrm{A}_{\mathrm{Acc}}$ quelqu'un " J'entends / écoute quelqu'un. »

\footnotetext{
${ }^{6}$ Notons qu'en espagnol la distinction singulier - pluriel semble influencer le marquage différentiel de l'objet, en ce sens qu'il est plus facile de ne pas avoir la marque avec les objets directs au pluriel (Laca (c.p.)). Nous n'avons pas pu vérifier la pertinence de ce facteur (local) dans le cadre de cet article. Nous laissons cet aspect pour de futures recherches.

${ }^{7}$ Rappelons que nous nous intéressons à ce phénomène tel qu'il caractérise l'espagnol standard (c.-à-d. le dialecte castillan). Nous ne prendrons pas en compte les différences qui apparaissent dans les autres dialectes.
} 


\section{$A$ est optionnel avec}

I. Les GN spécifiques : noms propres [- animé] (21), GN définis [+ animé] (22)

(21) Conoce (a) Sevilla connais $\mathrm{A}_{\mathrm{Acc}}$ Séville « Je connais Séville.»

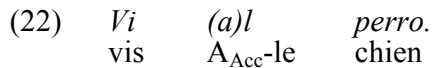
«J'ai vu le chien. »

\section{Les GN indéfinis non spécifiques [+ humain] :}

(23) a. Han matado (a) un buscador ont tué $\mathrm{A}_{\mathrm{Acc}}$ un chercheur "On a tué un chercheur. "

b. Busco (a) una cocinera. cherche $A_{\text {Acc }}$ une cuisinière « Je cherche une cuisinière. »

\section{$A$ est exclu avec :}

\section{Les pronoms déictiques et anaphoriques [- humain] :}

(24) Escucho $(* a) l$ mío. entends $\mathrm{A}_{\mathrm{Acc}}-\mathrm{le}$ mien

$($ el mio $=$ mon perroquet, mon $\mathrm{CD})$ « J'entends / écoute le mien. »

II. Les GN définis spécifiques [- animé] :

(25) Compré (*a) la casa. achetai $\mathrm{A}_{\mathrm{Acc}}$ la maison

« J'ai acheté la maison. »

III. Les GN non spécifiques : GN indéfinis [- humain] (26), noms nus [+/humain] (27)

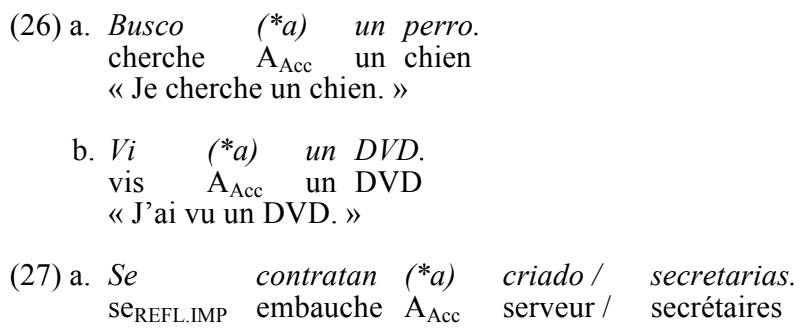


« On embauche (un) serveur / des secrétaires. »

b. Busco (*a) libros.

cherche $\mathrm{A}_{\mathrm{Acc}}$ livres

«Je cherche des livres. »

\section{Les quantifieurs nus [- humain] :}

$\begin{array}{lll}\text { (28) No he visto }\left(^{*} a\right) & \text { nada. } \\ \text { ne ai vu A } & \text { rien } \\ \text { «Je n'ai rien vu. » } & \end{array}$

\section{Les GN génériques [+/- animé] :}

(29) a. Respetan (*a) las chicas. respectent $A_{A c c}$ les filles «Ils / elles respectent les filles.»

b. Adoran (*a) los perros. adorent $\mathrm{A}_{\mathrm{Acc}}$ les chiens «Ils / elles adorent les chiens.»

c. Adoran (*a) las novelas. adorent $\mathrm{A}_{\mathrm{Acc}}$ les romans «Ils / elles adorent les romans. »

\subsubsection{Le sarde}

Les données du sarde nous ont été fournies par des locuteurs natifs des dialectes logoudorien et campidanien, qui sont parlés dans le centre-nord de la Sardaigne. Certaines d'entre elles sont adaptées de Bossong (1985), Blasco Ferrer (1986, c.p.), Jones (1993), Floricic (2003), Mensching (1994, 2005), Blasco Ferrer \& Ingrassia (2007), Molinu (c.p.).

\section{$A$ est obligatoire avec les objets directs réalisés comme:}

I. Pronoms [+ humain] : personnels toniques (30), déictiques et anaphoriques (31)

(30) Appo vistu solu *(a) isse. ai vu seulement $A_{\text {Acc }}$ lui « Je n'ai vu que lui. »

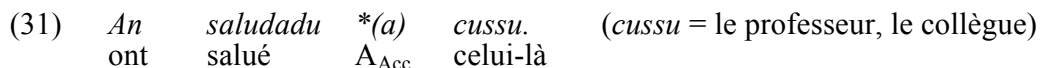
«Ils / elles ont salué celui-là. »

II. Noms propres [+/- animé] (32) et GN définis assimilables aux noms propres [+ humain] (33) : 
(32) Appo vistu *(a) Napoli. ai vu $\mathrm{A}_{\mathrm{Acc}}$ Naples «J'ai vu Naples. »

(33) Appo vistu *(a) babbu. ai vu $A_{\text {Acc }}$ grand-père « J'ai vu (mon) grand-père. »

III. Les quantifieurs nus [+ humain] :
(34) No appo vistu *(a) nesciune. ne ai vu $\mathrm{A}_{\mathrm{Acc}}$ personne « Je n'ai vu personne. »

\section{$A$ est optionnel avec :}

\section{Les GN définis spécifiques [+ humain] :}

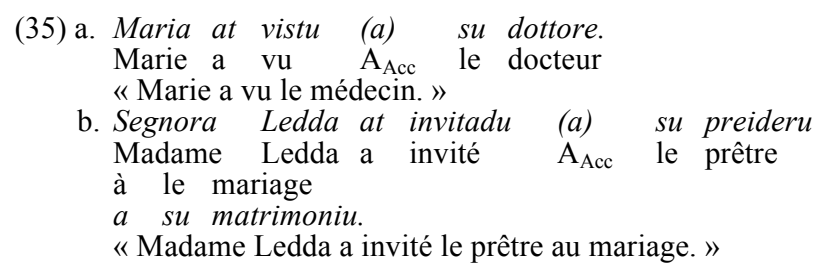

\section{$A$ est exclu avec:}

I. Les pronoms déictiques et anaphoriques [- humain] :
(36) $\quad(* A) \quad$ cale keres comporare? (cale = le cheval, le livre) $\mathrm{A}_{\text {Acc }}$ lequel veux acheter «Lequel veux-tu acheter?»

\section{Les GN définis spécifiques [- humain] :}

(37) Appo vistu $\left({ }^{*} a\right)$ su cane. « J'ai vu le chien. »

III. Les GN non spécifiques [+/- humain] : GN indéfinis (38), noms nus (39)

(38) Eris an salidadu (*a) unu pastore. hier ont salué $\mathrm{A}_{\mathrm{Acc}}$ un berger «Hier, ils / elles ont salué un berger. »

(39) An assessinatu $\quad(* a) \quad \begin{aligned} & \text { sordatos. } \\ & \text { soldats }\end{aligned}$ 
«Ils / elles ont assassiné des soldats. »

\section{Les quantifieurs nus [- humain] :}

(40) No appo vistu $\left({ }^{*} a\right)$ nudda. ne ai vu $\mathrm{A}_{\mathrm{Acc}}$ rien

« Je n'ai rien vu. »

\section{Les GN génériques [+/- animé] :}

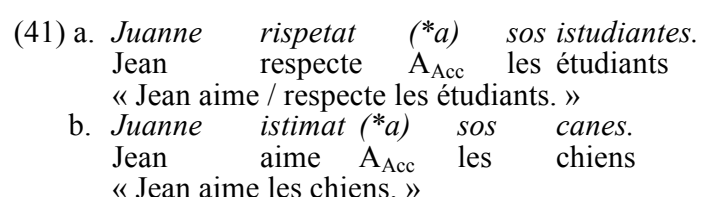

\subsubsection{Synthèse}

Les données examinées précédemment confirment les prédictions des échelles faites au début de la section. C'est-à-dire que le marquage différentiel est obligatoire dans les trois langues pour les objets qui occupent une position haute dans la hiérarchie des paramètres locaux. Il s'agit plus spécifiquement des objets directs réalisés comme pronoms personnels, pronoms déictiques et anaphoriques à référent humain ou encore comme noms propres à référent animé. Inversement, le marquage est exclu dans les trois langues pour les objets qui occupent une position basse dans la hiérarchie. Ce sont les objets directs réalisés comme groupes nominaux non spécifiques (plus précisément, comme noms nus, quelle que soit la nature de leur référent, ou comme groupes nominaux indéfinis à référent non humain). De même, les objets directs réalisés comme groupes nominaux spécifiques à référent inanimé ou comme groupes nominaux définis en lecture générique ne sont marqués dans aucune des langues examinées.

Dès lors que l'on quitte les pôles des hiérarchies pour les positions intermédiaires, on constate que le traitement du MDO n'est pas homogène dans les langues étudiées ici. Autrement dit, il existe de nombreux cas de variation entre les trois langues, que nous détaillerons dans la section 3 ci-dessous.

\subsection{Les paramètres globaux}

Dans cette section, nous commençons la présentation d'une nouvelle série de données, que nous décrirons en fonction des paramètres globaux. Rappelons que ces derniers ont souvent été corrélés au caractère topicalisé de l'objet direct. Pour le phénomène en question ici, le terme «topicalisé » est habituellement employé dans un sens très large, pour désigner les objets directs dont le référent est saillant (c.-à-d. proéminent) dans le discours (cf. aussi Leonetti (2003), Landragin (2007)). 
Ainsi, il est communément admis que l'échelle de topicalisation comporte les valeurs $[+]$ et $[-]$, correspondant respectivement à des effets de topicalisation et de non topicalisation. En réalité, les choses sont plus compliquées que cette dichotomie le laisse entendre. Comme nous pourrons le constater dans ce qui suit, la valeur [+ topicalisé] peut être induite par des facteurs de natures différentes. Nous nous intéressons principalement aux facteurs suivants : (i) la nature lexicale du verbe dont dépend l'objet direct, (ii) l'interprétation du sujet, (iii) la dislocation à gauche (qui est accompagnée, dans la plupart des cas, d'une accentuation et / ou d'une prosodie spéciale/s), ou encore (iv) la modification de l'objet ${ }^{8}$.

Il est important de souligner que ces facteurs agissent dans la plupart des cas sur des objets sur lesquels les facteurs locaux n'ont pas d'incidence (cf. la section 2.1 ci-dessus). Autrement dit, les facteurs globaux interagissent avec les facteurs locaux et c'est pourquoi on peut aboutir à des situations de marquage inattendu. Plus précisément, les objets qui excluent ou n'acceptent qu'optionnellement le marquage différentiel du fait qu'ils occupent une position basse ou intermédiaire dans la hiérarchie des paramètres locaux peuvent néanmoins recevoir la marque de façon optionnelle ou obligatoire sous l'influence de l'un des facteurs globaux mentionnés précédemment. Comme nous le verrons dans ce qui suit, c'est notamment le cas de l'espagnol et moins souvent du roumain et du sarde.

\subsubsection{Les propriétés lexicales du verbe}

Les études sur le marquage différentiel de l'objet en espagnol ont montré que les propriétés lexicales du verbe peuvent jouer un rôle dans l'apparition de la marque. Plus précisément, il s'agit des propriétés de sélection du verbe par rapport au caractère animé ou inanimé de l'objet. De ce point de vue, on admet qu'il existe trois classes de verbes (cf. l'article sur l'espagnol de von Heusinger \& Kaiser (2007)) :

$\begin{array}{lll}\text { classe 1 } & \text { classe 2 } & \text { classe } 3 \\ \text { V à OD [+ animé }] & \text { V à OD [+/- animé] } & \text { V à OD }[(+/-) /- \text { animé }] \\ \text { herir « blesser » } & \text { ver « voir » } & \text { poner « mettre » } \\ \text { matar « tuer » } & \text { hallar « trouver » } & \text { tomar " prendre » }\end{array}$

On considère que les verbes de la classe 1 se combinent exclusivement avec des objets directs à référent animé et, de ce fait, ont tendance à imposer la marque à tous les objets de ce type, quelle que soit leur interprétation (indéfinie et / ou non spécifique). Les exemples ci-dessous, qui comportent des objets indéfinis non spécifiques ayant des référents animés, illustrent ce propos :

\footnotetext{
${ }^{8}$ Pour d'autres facteurs globaux pouvant influencer le marquage différentiel de l'objet, comme l'anaphoricité ou la coordination, le lecteur peut se rapporter aux études sur l'espagnol de Laca $(2002,2006)$.
} 


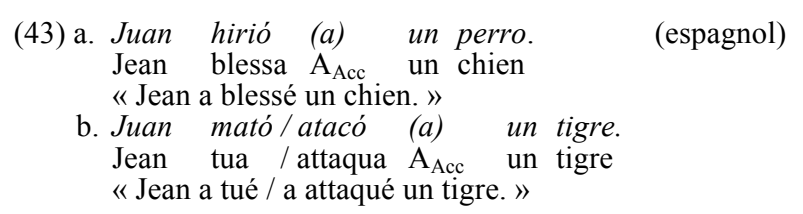

En revanche, lorsque les objets du type donné en (43) se combinent avec des verbes appartenant aux classes 2 et 3 ci-dessus, le marquage est exclu, comme dans les exemples suivants :

$$
\begin{aligned}
& \text { (44) a. Juan ha visto }\left({ }^{*} a\right) \text { un tigre. (espagnol) } \\
& \text { Jean a vu } \mathrm{A}_{\mathrm{Acc}} \text { un tigre } \\
& \text { "Jean a vu un tigre." } \\
& \text { b. Juan tomará también }\left({ }^{*} a\right) \text { un perro para guardia. } \\
& \text { Jean prendra aussi } \mathrm{A}_{\mathrm{Acc}} \text { un chien pour gardien } \\
& \text { "Jean prendra aussi un chien comme gardien." }
\end{aligned}
$$

Le MDO en roumain et en sarde n'est pas sensible aux propriétés lexicales du verbe. Les exemples suivants, qui sont similaires à ceux donnés en (43) cidessus, montrent que les object directs animés indéfinis non spécifiques à référent animé ne sont pas marqués par pe et $a$, respectivement, quand ils se combinent avec des verbes de la classe 1 :

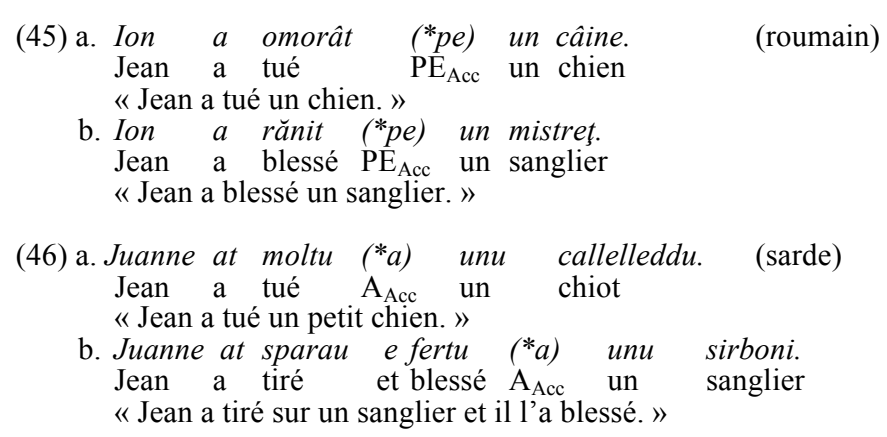

\subsubsection{L'interprétation du sujet ${ }^{9}$}

Les effets de cette contrainte sont visibles avec les objets directs réalisés comme groupes nominaux indéfinis à référent humain ou comme groupes nominaux définis spécifiques à référent inanimé.

Lorsque ces objets apparaissent avec des verbes dont le sujet est interprété comme Agent ou Cause, ils sont obligatoirement marqués si leur référent est

\footnotetext{
${ }^{9}$ Cette contrainte a été mise en évidence par Torrego Salcedo (1999), qui l'appelle « la contrainte d'agentivité ».
} 
animé (47a), alors qu'ils ne le sont qu'optionnellement si leur référent est inanimé $(47 \mathrm{~b}-\mathrm{c})$. Notons que cette contrainte vaut pour l'espagnol :

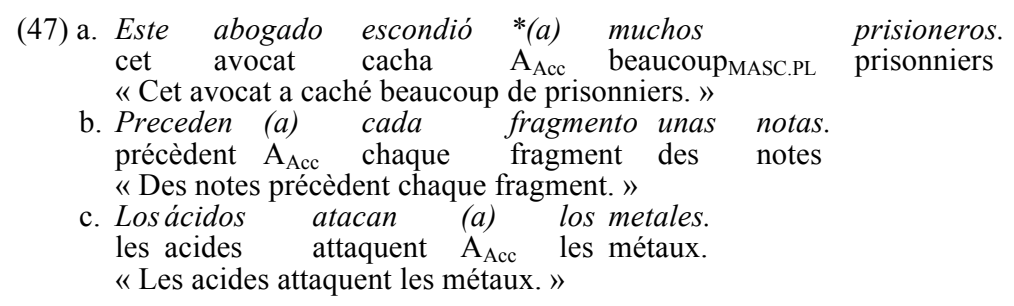

En revanche, si le sujet de la phrase n'est pas interprété comme Agent ou Cause, le MDO est exclu, quelle que soit la nature du référent de l'objet, comme dans les exemples ci-dessous :

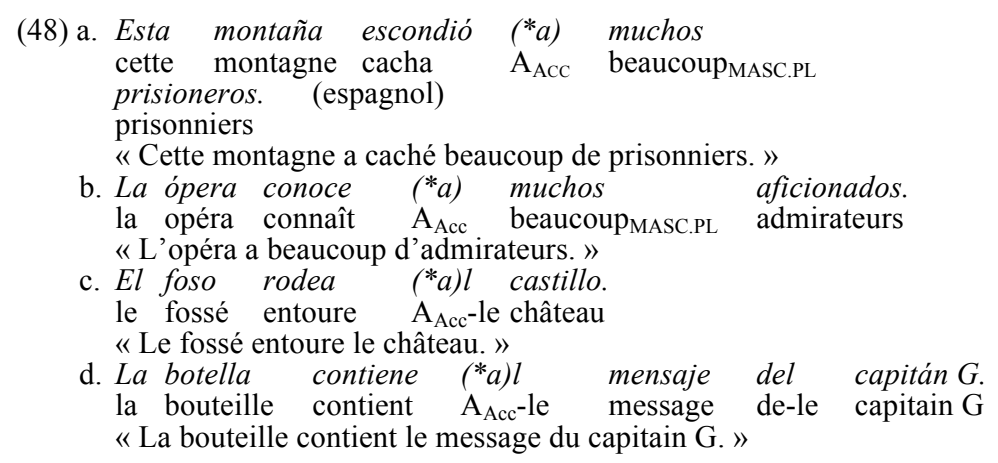

En revanche, la contrainte en cause ne s'applique pas au roumain et au sarde. Comparons en ce sens les exemples espagnols en (47) avec les exemples roumains et sardes donnés en (49) et (50) respectivement :

(49) a. Acest avocat (i-)a ascuns (pe) mulţi prizonieri. (roumain, mêmes traductions que (47))

b. Câteva note precedă (*pe) fiecare fragment.

c. Acizii atacă $\left({ }^{*}\right.$ pe) metale.

(50) a. Sus res an assessinatu (*a) metas sordatos. (sarde) les rois ont assassiné $\mathrm{A}_{\mathrm{ACC}}$ nombreux soldats

«Les rois ont tué beaucoup de soldats. »

b. Sos pudzones saludan (*a) s'albore. les oiseaux saluent $A_{A C C}$ la aurore " Les oiseaux saluent l'aurore. »

c. Sos atzidos attacan (*a) sos ferros. les acides attaquent $\mathrm{A}_{\mathrm{ACC}}$ les métaux « Les acides attaquent les métaux. » 
Comme on peut donc le constater dans les exemples ci-dessus, le marquage différentiel en roumain et en sarde n'est pas sensible à l'interprétation du sujet. Malgré l'interprétation appropriée des sujets, comme Agent ou Cause, les objets directs des phrases données en (49) et (50), qui sont similaires à celles de l'espagnol, ne peuvent pas être précédés de pe ou de $a$. Notons que l'emploi facultatif de pe en (49a) n'est pas dû à l'interprétation du sujet, mais à d'autres facteurs (c.-à-d. la présence du quantifieur mulţi « nombreux / beaucoup » et le caractère animé humain de l'objet direct).

\subsubsection{La dislocation à gauche}

Un autre facteur global qui peut influencer le marquage différentiel de l'objet est représenté par la dislocation à gauche, plus précisément en position préverbale.

En ce qui concerne l'espagnol, ce facteur agit dans des contextes similaires à ceux mentionnés dans la section précédente, c'est-à-dire avec les objets directs réalisés comme groupes nominaux indéfinis non spécifiques à référent humain ou comme groupes nominaux définis spécifiques à référent inanimé. Rappelons que, selon la hiérarchie des paramètres locaux, les premiers sont optionnellement marqués, comme en (51a) ci-dessous, tandis que les seconds n'entraînent pas le marquage, comme en (51b) ci-dessous :

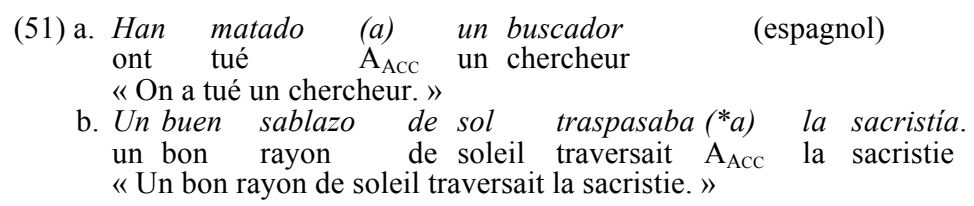

En revanche, la situation est différente lorsque les objets en question sont disloqués en position préverbale. C'est-à-dire que le marquage devient obligatoire pour les objets directs réalisés comme groupes nominaux indéfinis à référent humain $(52 a)$, alors qu'il est optionnel pour les objets réalisés comme groupes nominaux définis spécifiques à référent inanimé $(52 b)$ :

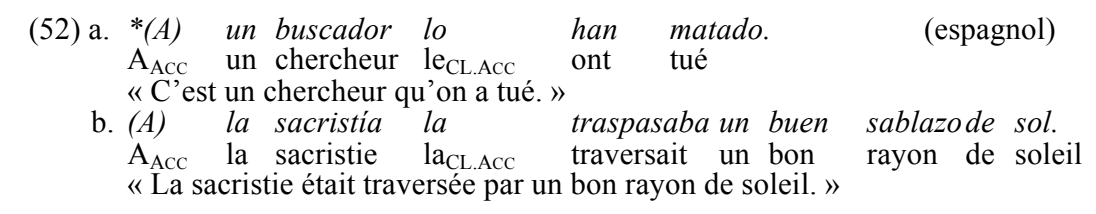

Notons que l'objet direct indéfini de (52a) acquiert une lecture spécifique.

En ce qui concerne le roumain, l'influence de ce facteur est plus restreinte, en ce sens qu'il ne rend le marquage obligatoire que dans le cas d'objets directs réalisés comme groupes nominaux définis spécifiques à référent humain. Selon la hiérarchie des facteurs locaux, ces derniers sont marqués optionnellement, comme dans l'exemple suivant : 


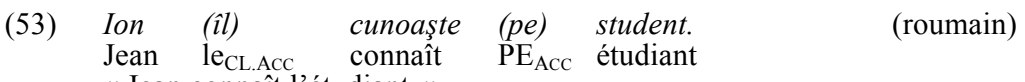
« Jean connaît l'étudiant. »

Lorsqu'ils apparaissent en position disloquée à gauche, les objets de ce type entraînent le marquage obligatoire :

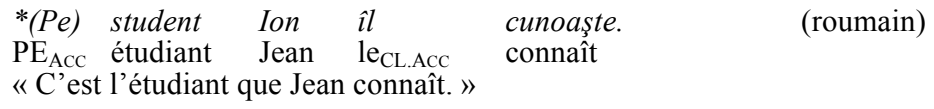

À la différence de l'espagnol, le roumain marque de façon optionnelle les objets directs réalisés par des groupes nominaux indéfinis à référent humain (55a), tandis qu'il rejette le marquage des objets directs réalisés comme groupes nominaux définis spécifiques à référent inanimé. Comparons les exemples roumains en (55) avec ceux de l'espagnol donnés en (52) ci-dessus :

(55) a. (Pe) un cercetător l-au omorât. (roumain, mêmes traductions que (52)) b. ( $\left.{ }^{*} P e\right)$ altar îl străbate o rază de soare.

Toujours concernant le roumain, il existe deux cas de marquage obligatoire des objets inanimés disloqués à gauche, qui semblent venir à l'encontre de l'impossibilité illustrée en (55b) ci-dessus. Le premier est représenté par l'exemple suivant, emprunté à la GLR (1966) :

$$
\begin{aligned}
& \text { *(Pe) trandafir l-a lăsat albina la urmă. (roumain) } \\
& \text { PE-ACC rose le } \text { CL.ACC}^{-a} \text { laisse abeille-la à fin } \\
& \text { «C'est la rose que l'abeille a laissée pour la fin. » }
\end{aligned}
$$

Comme on peut le constater, en (56), l'objet direct trandafir « rose » doit être précédé de $p e$. En réalité, le caractère obligatoire de la marque est dû au fait qu'il s'agit d'une personnification. Notons que l'exemple est tiré d'un conte de fées où les personnages se voient attribuer des traits humains. C'est ce qui rend obligatoire la présence de la marque.

Le second cas est représenté par les exemples ci-dessous :

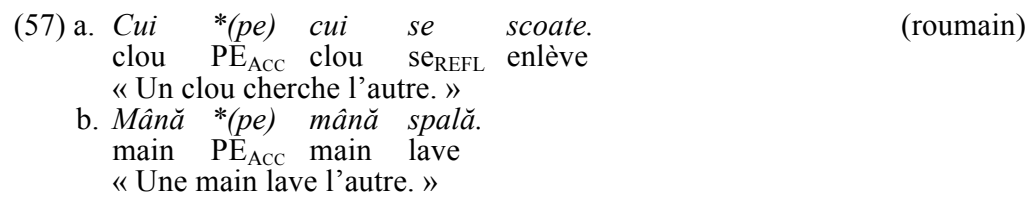

Il faut d'abord noter qu'en (57), il s'agit de proverbes, c'est-à-dire d'expressions figées. En outre, nous avons affaire à des constructions dans lesquelles il s'agit des mêmes lexèmes (i.e., cui « clou » et mînă « main ») qui sont contigus. Par ailleurs, les constructions en questions sont ambiguës, en ce sens que le sujet, l'objet direct et le verbe renvoient formellement à la même 
personne grammaticale. Comme nous le verrons dans la section 4.1.1 ci-dessous, la présence de pe est requise afin d'enlever l'ambiguïté fonctionnelle qui caractérise les éléments de la phrase. Le marquage obligatoire n'est donc pas dû à la dislocation en position préverbale.

Enfin, pour ce qui est du sarde, l'influence du facteur en question est quasisimilaire à ce qui se passe en roumain. Plus précisément, le sarde marque de façon obligatoire seulement les objets directs réalisés comme groupes nominaux définis spécifiques à référent humain lorsqu'ils se trouvent en position disloquée à gauche. Les exemples suivants illustrent ce propos :

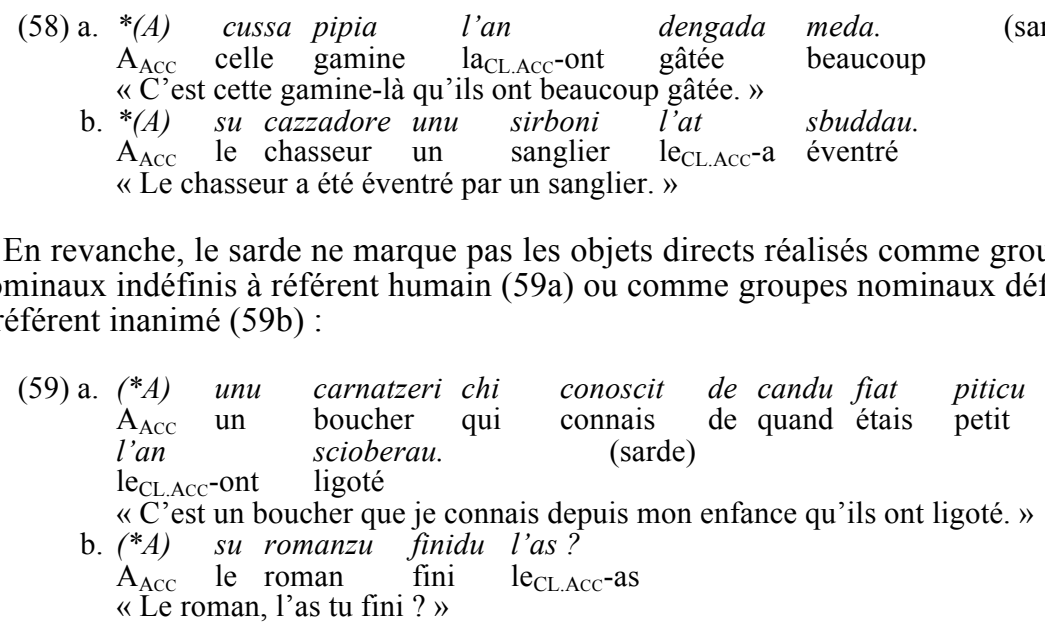

\subsubsection{Rôle des modifieurs de l'objet}

Notons enfin un quatrième facteur global qui peut intervenir dans le marquage différentiel de l'objet : la présence d'un modifieur.

Cette contrainte est pertinente pour les constructions dans lesquelles l'objet direct est réalisé comme pluriel nu à référent humain, comme groupe nominal indéfini à référent animé ou encore comme groupe nominal défini à référent animé en lecture générique. Selon la hiérarchie des paramètres locaux, rappelons que seuls les objets directs réalisés comme groupes nominaux indéfinis à référent humain, en espagnol, sont optionnellement marqués (cf. les exemples (23) cidessus et (60b-b') ci-dessous), tandis que tous les autres types d'objet que nous venons de mentionner rejettent le marquage. Nous rappelons brièvement cette impossibilité pour chacune des trois langues en cause :

\footnotetext{
(60) a. Detuvieron $\quad\left({ }^{*} a\right)$ hinchas. (pluriel nu) (espagnol) arrêtèrent $\quad \mathrm{A}_{\mathrm{ACC}}$ supporters " Ils / elles ont arrêté des supporters. "
} 


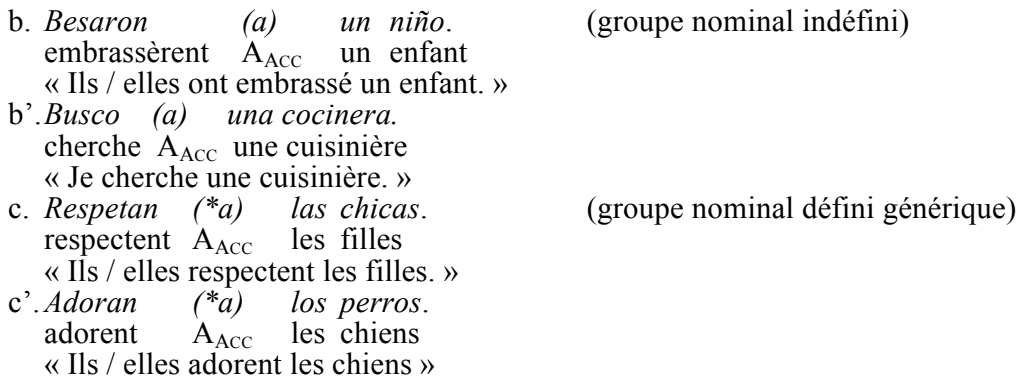

(61) a. Au arestat (*pe) suporteri. (pluriel nu) (roumain, mêmes traductions que (60))

b. Au sărutat (*pe) un copil.

b'. Caut (*pe) o bucătăreasă.

c. Respectă (*pe) fete.

c'. Adoră (*pe) câini. $\begin{array}{llll}\text { (62) a. An } & \text { moltu } & (* a) & \text { sordatos. } \\ \text { ont } & \text { tué } & \mathrm{A}_{\mathrm{AcC}} & \text { soldates }\end{array}$

(groupe nominal défini générique) " On a tué des soldats. »

b. An assessinatu (*a) ипи ont assassiné $\quad \mathrm{A}_{\mathrm{AcC}} \quad$ un "On a assassiné un soldat. »

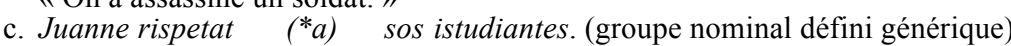
Jean respecte $A_{A C C}$ les étudiants

«Jean respecte les étudiants. »

$c^{\prime}$. Juanne istimat $\left({ }^{*} a\right)$ sos canes.

Jean aime $A_{A C C}$ les chiens

« Jean aime les chiens. »

En revanche, lorsque ces mêmes objets comportent des modifieurs, le marquage devient optionnel ou obligatoire, suivant les langues et le type d'objet.

Par exemple, en espagnol, les pluriels nus à référent humain entraînent le marquage optionnel lorsqu'ils sont modifiés. Les exemples suivants, tirés de Leonetti (2003), illustrent ce propos :

(63) a. Detuvieron (a) hinchas peligrosos del Atlético. (espagnol) arrêtèrent $\mathrm{A}_{\mathrm{AcC}}$ supporters dangereux de-le Atlético «Ils / elles ont arrêté des supporters dangereux de Atlético »

b. Conocemos (a) profesores que se pasan connaissons $\mathrm{A}_{\mathrm{ACC}}$ professeurs qui se $\mathrm{REFL}_{\text {passent }}$ el fin de semana trabajando. le fin de semaine travaillant

« Nous connaissons des professeurs qui passent leur fin de semaine à travailler. »

Les objets directs de l'espagnol réalisés comme groupes nominaux indéfinis à référent humain entraînent le marquage obligatoire, quand ils sont accompagnés 
de modifieurs (64). Soulignons que les objets en question donnent lieu à une lecture spécifique. Les exemples suivants sont adaptés de Torrego Salcedo (1999) :

$$
\begin{aligned}
& \begin{array}{llll}
\text { (64) a. Besaron } & *(a) & \text { un niño llorando. } \\
\text { embrassèrent } & \mathrm{A}_{\mathrm{AcC}} & \text { un enfant criant }
\end{array} \\
& \text { " Ils / elles ont embrassé un enfant qui pleurait. » } \\
& \text { b. Busco *(a) una cocinera que sabe inglés }{ }^{10} \text {. } \\
& \text { " Je cherche une cuisinière qui parle l'anglais. " }
\end{aligned}
$$

Si le référent d'un groupe nominal indéfini à fonction d'objet direct modifié est animé (c.-à-d. non humain), alors le marquage n'est qu'optionnel :
(65) Busco (a) un perro con patas blancas. (espagnol) cherche $A_{\text {ACC }}$ un chien avec pattes blanches « Je cherche un chien ayant les pattes blanches.»

Les objets directs de l'espagnol réalisés comme groupes nominaux définis à référent animé, en lecture générique, sont marqués de façon optionnelle, lorsqu'ils sont modifiés :
(66) a. Respetan (a) las chicas inteligentes / rubias. (espagnol) respectent $\mathrm{A}_{\mathrm{ACC}}$ les filles intelligentes / blondes «Ils / elles respectent les filles intelligentes / blondes. »
b. Adoran (a) los perros con patas blancas. adorent $\mathrm{A}_{\mathrm{ACC}}$ les chiens avec pattes blanches « Ils / elles adorent les chiens ayant les pattes blanches. »

En ce qui concerne le roumain, l'influence de la modification sur le marquage différentiel de l'objet peut être décrite de la façon suivante.

Les pluriels nus à référent humain ayant la fonction d'objet direct n'entraînent pas le marquage lorsqu'ils sont modifiés :

(67) a. Au arestat (*pe) suporteri periculoşi ai Atleticului. (roumain, mêmes traductions que (63))

b. Cunoaştem ( *pe) profesori care îşi petrec sfârşitul de săptămână lucrând.

Les objets directs réalisés comme groupes nominaux indéfinis à référent humain entraînent le marquage optionnel, quand ils sont accompagnés de modifieurs (68). Tout comme en espagnol, soulignons que les objets en question donnent lieu à une lecture spécifique :

\footnotetext{
${ }^{10}$ Notons que le marquage est optionnel dans un exemple comme (64b) si le verbe de la phrase relative est au subjonctif: Busco (a) una cocinera que sepa inglés cherche $\mathrm{A}_{\mathrm{Acc}}$ une cuisinière qui sache anglais "Je cherche une cuisinière qui connaisse l'anglais". Dans ce cas, l'interprétation de l'objet direct n'est pas spécifique.
} 
(68) a. (L-)au sărutat (pe) un copil care plângea. (roumain, mêmes traductions que (64))

b. (O) caut (pe) o bucătăreasă care ştie engleză ${ }^{11}$.

En revanche, les objets directs réalisés comme groupes nominaux indéfinis à référent non humain excluent le marquage, même s'ils sont accompagnés d'un modifieur :

(69) Caut (*pe) un câine cu labele albe. (roumain, même traduction que (65))

Les groupes nominaux définis du roumain, en fonction d'objet direct générique, se comportent de la façon suivante, par rapport à la modification : (i) s'ils renvoient à des référents humains, ils sont marqués de façon optionnelle (70a); (ii) s’ils renvoient à des référents non humains, ils ne peuvent pas être marqués $(70 \mathrm{~b})$ :

(70) a. (Le) respectă (pe) fetele inteligente / blonde. (roumain, mêmes traductions que (66))

b. Adoră (*pe) câinii cu labele albe.

Pour ce qui est du sarde, la présence d'un modifieur avec les types d'objet en question n'est pas pertinente pour le marquage différentiel. C'est-à-dire que la marque est exclue dans chacun des trois cas illustrés ici pour l'espagnol et le roumain.

L'exemple (71) illustre ce propos pour les pluriels nus à référent humain quand ils sont modifiés, tandis que les exemples (72) et (73) l'illustrent respectivement pour les groupes nominaux indéfinis à référent humain et pour les groupes nominaux définis à référent animé en lecture générique :

(71) An moltu $\left({ }^{*} a\right)$ sordatos intelligentes. (sarde) ont tué $\mathrm{A}_{\mathrm{ACC}}$ soldates intelligents «On a tué des soldats intelligents. »

(72) a. An assessinatu (*a) unu sordato ki appo acciappadu ont assassiné $\mathrm{A}_{\mathrm{ACC}}$ un soldat que ai aperçu custu madzanu.

"On a assassiné un soldat que j'ai aperçu ce matin. »

b. Appo invitadu (*a) una pipia dengada. ai invité $\mathrm{A}_{\mathrm{ACC}}$ une gamine gâté «J'ai invité une gamine gâtée. »

${ }^{11}$ À la différence de ce qui se passe en espagnol, notons qu'en roumain le marquage est exclu dans un exemple comme (68b) si le verbe de la phrase relative est au subjonctif: Caut (*pe) o bucătăreasă care să ştie engleză cherche $\mathrm{A}_{\mathrm{Acc}}$ une cuisinière SUBJ sait anglais « Je cherche une cuisinière qui connaisse l'anglais ». Dans ce cas, l'interprétation de l'objet direct n'est pas spécifique. 
(73) a. Juanne rispetat (*a) sos istudiantes intelligentes. Jean respecte $\mathrm{A}_{\mathrm{ACC}}$ les étudiants intelligents

(sarde) « Jean respecte les étudiants intelligents. »

b. Juanne istimat $\left({ }^{*}\right.$ a) sos canes de cursa. Jean aime $\mathrm{A}_{\mathrm{ACC}}$ les chiens de course " Jean aime les chiens de course. »

\subsubsection{Synthèse}

Pour résumer ce qui a été dit dans cette section : nous avons donné des exemples pour démontrer qu'il existe une série de facteurs globaux qui favorisent le marquage différentiel de l'objet.

Nous avons constaté que ces facteurs sont pertinents seulement pour certains types d'objet direct. Il s'agit notamment des objets occupant des positions basses dans la hiérarchie des paramètres locaux, en l'occurrence les pluriels nus à référent humain, les groupes nominaux indéfinis à référent animé et les groupes nominaux définis à référent animé en lecture générique.

Nous pouvons affirmer que les paramètres globaux ont un réel impact dans la mesure où ils annulent les paramètres locaux, du fait que les premiers entraînent le marquage des objets qui ne sont pas a priori censés se combiner avec la marque.

Nous avons également constaté que le poids des paramètres globaux est plus important en espagnol qu'en roumain et en sarde. C'est-à-dire que le marquage différentiel de l'objet en espagnol est sensible à tous les facteurs examinés, tandis qu'en roumain le marquage est partiellement sensible à la dislocation en position préverbale et à la présence d'un modifieur. Le sarde est encore moins sensible aux paramètres globaux, en ce sens que le marquage différentiel n'est influencé que par la dislocation en position préverbale. Ce point de variation représente d'ailleurs la différence majeure entre les trois langues mentionnées, séparant l'espagnol, d'une part, du roumain et du sarde, d'autre part. Nous reviendrons sur cet aspect dans la section suivante.

\section{INTERPRETATION DES RESULTATS}

Les tableaux suivants reprennent brièvement les principaux résultats des descriptions effectuées précédemment. Les trois premiers renvoient à la description des données selon les paramètres locaux, tandis que les deux derniers renvoient aux données décrites selon les paramètres globaux.

Les symboles «+», «-» et « \pm » désignent les constructions dans lesquelles le marquage différentiel de l'objet est obligatoire, exclu ou optionnel, respectivement. Le symbole « $\varnothing$ » renvoie au fait que la propriété en question n'est pas pertinente pour le marquage différentiel de l'objet. 
Tableau 1

\begin{tabular}{|c|c|c|c|c|}
\hline & \multicolumn{4}{|c|}{ Pronoms } \\
\hline & \multicolumn{3}{|c|}{ personnels toniques } & \multicolumn{2}{c|}{ déictiques et anaphoriques } \\
\hline & humain & in-/animé & humain & in-/animé \\
\hline $\mathbf{R}$ & + & $\varnothing$ & + & + \\
\hline $\mathbf{E}$ & + & $\varnothing$ & + & - \\
\hline S & + & $\varnothing$ & + & - \\
\hline
\end{tabular}

Tableau 2

\begin{tabular}{|c|c|c|c|c|c|c|c|}
\hline & \multicolumn{2}{|c|}{$\begin{array}{c}\text { Noms propres et GN } \\
\text { définis assimilables } \\
\text { aux noms propres }\end{array}$} & \multicolumn{2}{|c|}{ GN définis spécifiques } & \multicolumn{2}{c|}{ Quantifieurs nus } \\
\cline { 2 - 9 } & animé & inanimé & humain & animé & inanimé & humain & inanimé \\
\hline R & + & - & \pm & - & - & + & - \\
\hline E & + & \pm & + & \pm & - & + & - \\
\hline S & + & + & \pm & - & - & + & - \\
\hline
\end{tabular}

Tableau 3

\begin{tabular}{|c|c|c|c|c|c|}
\hline & \multicolumn{4}{|c|}{ GN non spécifiques } & \multirow{3}{*}{$\begin{array}{c}\text { GN génériques } \\
\text { humain / in-/animé }\end{array}$} \\
\hline & \multicolumn{3}{|c|}{ GN indéfinis } & \multirow{2}{*}{$\begin{array}{l}\text { Noms nus } \\
\text { humain / in-/animé }\end{array}$} & \\
\hline & humain & animé & inanimé & & \\
\hline $\mathbf{R}$ & - & - & - & - & - \\
\hline $\mathbf{E}$ & \pm & - & - & - & - \\
\hline $\mathbf{S}$ & - & - & - & - & - \\
\hline
\end{tabular}

Tableau 4

\begin{tabular}{|c|c|c|c|}
\hline & $\begin{array}{c}\text { Propriétés lexicales du } \\
\text { verbe }\end{array}$ & \multicolumn{2}{|c|}{ Interprétation du sujet } \\
\cline { 3 - 4 } & GN indéfinis humains & $\begin{array}{c}\text { GN définis spécifiques } \\
\text { inanimés }\end{array}$ \\
\hline R & - & - & - \\
\hline E & + & + & \pm \\
\hline S & - & - & - \\
\hline
\end{tabular}

Tableau 5

\begin{tabular}{|c|c|c|c|c|c|c|c|c|}
\hline & \multicolumn{3}{|c|}{ Dislocation à gauche } & \multicolumn{5}{|c|}{ Modification } \\
\hline & \multirow{2}{*}{$\begin{array}{l}\text { GN } \\
\text { indéfinis } \\
\text { humains }\end{array}$} & \multicolumn{2}{|c|}{$\begin{array}{l}\text { GN définis } \\
\text { spécifiques }\end{array}$} & \multirow{2}{*}{$\begin{array}{c}\text { Pluriels } \\
\text { nus } \\
\text { humains }\end{array}$} & \multicolumn{2}{|c|}{ GN indéfinis } & \multicolumn{2}{|c|}{ GN génériques } \\
\hline & & humain & $\begin{array}{c}\text { in-/ } \\
\text { animé }\end{array}$ & & humain & animé & humain & animé \\
\hline $\mathbf{R}$ & \pm & + & - & - & \pm & - & \pm & - \\
\hline $\mathbf{E}$ & + & + & \pm & \pm & + & \pm & \pm & \pm \\
\hline $\mathbf{S}$ & - & + & - & - & - & - & - & - \\
\hline
\end{tabular}


Les résultats enregistrés dans les tableaux ci-dessus appellent quelques commentaires.

En premier lieu, soulignons une fois de plus que le marquage différentiel de l'objet dans les langues romanes confirme les prédictions que nous avons faites sur la base des échelles décrites au début de la section $\mathbf{2}$ ci-dessus. Autrement dit, les données des trois langues en question ont montré qu'un objet direct qui occupe une position supérieure sur l'échelle pertinente a plus de chances d'être marqué (cf. le premier tableau ci-dessous) qu'un objet qui occupe une position inférieure sur la même échelle (cf. le troisième tableau ci-dessous).

En second lieu, notons un autre point intéressant qui concerne la variation à travers les langues romanes. Dans cette optique, on observe deux types de variation: (i) une variation forte et (ii) une variation faible. Dans ce qui suit, nous ferons quelques remarques sur ce dernier point.

\subsection{La variation forte}

La variation forte concerne pour la plupart du temps les objets directs qui tombent sous l'incidence de certains facteurs globaux (voir le Tableau 4 cidessus). De façon moins systématique, elle concerne les propriétés inhérentes des objets directs (voir le Tableau 1 ci-dessus). La variation forte peut être exprimée comme suit :

(a) Le roumain n'est pas sensible au caractère $+/$ - animé de l'objet direct quand ce dernier est réalisé comme pronom déictique ou anaphorique, c'est-àdire que le roumain marque tous les pronoms déictiques ou anaphoriques en fonction d'objet direct quelle que soit la nature [+/- humain], [+/- animé] de leur référent (voir l'exemple (5)). En revanche, l'espagnol et le sarde sont sensibles à ce facteur et marquent seulement les pronoms dont le référent a le trait [+ humain] (comparer les exemples (17) et (31) aux exemples (24) et (36)). Notons que ce point de variation forte est le seul à concerner les propriétés inhérentes des objets directs ou, autrement dit, les paramètres locaux.

(b) L'espagnol est sensible aux propriétés lexicales du verbe avec lequel l'objet direct se combine, c'est-à-dire que l'espagnol marque de façon optionnelle les objets directs réalisés comme groupes nominaux indéfinis non spécifiques à référent animé, lorsqu'ils se combinent avec des verbes de la classe matar " tuer», herir "blesser», etc. (voir l'exemple (43)). Par contraste, le roumain et le sarde ne sont pas sensibles à ce facteur et, par conséquent, ne marquent pas les objets en question (voir les exemples (45) et (46)).

(c) L'espagnol est sensible à l'interprétation du sujet, c'est-à-dire que cette langue marque de façon obligatoire les objets directs réalisés comme groupes nominaux indéfinis à référent humain (voir l'exemple (47a)) et de façon optionnelle les objets directs réalisés comme groupes nominaux définis à référent inanimé (voir les exemples $(47 \mathrm{~b}-\mathrm{c})$ ), lorsque le sujet de la phrase est interprété 
comme Agent ou Cause. En revanche, le roumain et le sarde ne sont pas sensibles à ce facteur (voir les exemples (49) et (50)).

(d) L'espagnol est sensible à la présence des modifieurs avec les objets directs réalisés comme noms pluriels nus à référent humain et marque de façon optionnelle ce type d'objet (voir l'exemple (64)). En revanche, le roumain et le sarde ne marquent pas les pluriels nus lorsqu'ils sont accompagnés de modifieurs (voir les exemples (67) et (71)).

\subsection{La variation faible}

La variation faible concerne les objets directs occupant des positions situées vers le milieu de la hiérarchie des facteurs locaux (voir le Tableau 2 ci-dessus) ou encore les objets qui tombent sous l'incidence de certains facteurs globaux (voir le Tableau 5 ci-dessus). Elle peut être exprimée comme suit :

(a) Le roumain est sensible au caractère +/- animé du référent des objets directs réalisés comme noms propres, c'est-à-dire que le roumain marque uniquement les noms propres à référent animé (voir l'exemple (6)). Par contraste, l'espagnol marque de façon optionnelle les noms propres à référent inanimé ayant la fonction d'objet direct (voir l'exemple (21)), tandis que le sarde les marque tous de façon obligatoire (voir l'exemple (32)).

(b) L'espagnol n'est pas sensible au caractère +/- humain du référent des objets directs réalisés comme groupes nominaux définis, c'est-à-dire que l'espagnol marque obligatoirement les objets directs définis à référent humain (voir l'exemple (19)) et de façon optionnelle les objets directs définis à référent animé (voir l'exemple (22)). En revanche, le roumain et le sarde marquent de façon optionnelle les objets directs définis à référent humain (voir les exemples (9) et (35)), et ne marquent pas les objets directs définis à référent animé (voir les exemples (11) et (37)).

(c) L'espagnol n'est pas sensible au caractère $+/$ - spécifique des objets directs réalisés comme groupes nominaux indéfinis à référent humain, en ce sens que l'espagnol marque de façon optionnelle les objets en question (voir l'exemple (23)). Par contraste, le roumain et le sarde ne marquent pas les groupes nominaux indéfinis non spécifiques à référent humain (voir les exemples (12) et (38)).

(d) L'espagnol est plus sensible à la dislocation à gauche de certains types d'objet que ne le sont le roumain et le sarde. Plus précisément, l'espagnol marque obligatoirement les objets directs réalisés comme groupes nominaux indéfinis à référent humain quand ils sont disloqués à gauche (voir l'exemple (52a)), alors que le roumain les marque de façon optionnelle (voir l'exemple (55a)) et que le sarde ne les marque pas (voir l'exemple (59a)). De même, l'espagnol marque de façon optionnelle les objets directs réalisés comme 
groupes nominaux définis à référent inanimé, lorsqu'ils sont disloqués à gauche (voir l'exemple (52b)), tandis que le roumain et le sarde ne marquent pas ce type d'objet (voir les exemples (55b) et (59b)).

(e) L'espagnol est plus sensible à la présence des modifieurs accompagnant certains types d'objet direct que ne le sont le roumain et le sarde. C'est-à-dire que l'espagnol marque obligatoirement les objets directs réalisés comme groupes nominaux indéfinis à référent humain, lorsqu'ils comportent un modifieur (voir l'exemple (64)), tandis que le roumain les marque de façon optionnelle (voir l'exemple (68)) et que le sarde ne les marque pas (voir l'exemple (72)). De même, l'espagnol marque optionnellement les objets directs réalisés comme groupes nominaux indéfinis à référent animé, quand ils sont accompagnés d'un modifieur (voir l'exemple (65)), tandis que le roumain et le sarde ne marquent pas ce type d'objet (voir l'exemple (69)). Enfin, le sarde ne marque pas les objets directs réalisés comme groupes nominaux définis modifiés en lecture générique (voir l'exemple (73)), tandis que l'espagnol marque de façon optionnelle ceux qui ont un référent animé (voir l'exemple (66)) et le roumain uniquement ceux qui ont un référent humain (voir l'exemple (70a)).

\section{PRECEDENTES ANALYSES DU PHENOMENE}

Les contrastes observés ci-dessus ont constitué la base d'analyses variées et parfois controversées. Dans ce qui suit, nous reviendrons brièvement sur certaines de ces analyses. Soulignons d'emblée qu'elles ne se situent pas toutes sur le même plan, c'est-à-dire qu'elles n'essaient pas d'expliquer l'existence du phénomène dans son ensemble, mais seulement certains de ses aspects. C'est la raison pour laquelle nous les présentons comme appartenant à trois types d'approches :

(i) les analyses fonctionnalistes,

(ii) les analyses sémantico-lexicales,

(iii) les analyses en termes de facteurs multiples.

\subsection{Les analyses fonctionnalistes ${ }^{12}$}

Dans cette classe, on distingue traditionnellement deux analyses. La première qui sera présentée ici a été avancée pour le roumain et l'espagnol. La seconde concerne seulement l'espagnol.

${ }^{12}$ Parmi les auteurs qui soutiennent cette thèse, voir Diez (1876), Hills (1920), Lenz (1944), Puşcariu (1922), Meier (1947, 1948), Onu (1959), Niculescu (1959), GLR (1966), Guţu Romalo (1973), RAE (1973), Pană Dindelegan (1976, 1997, 1999), GALR (2005). 
4.1.1. La thèse de la désambiguïsation entre le sujet et l'objet direct

L'argument principal de cette thèse est que le marquage permet d'éviter une « confusion » possible entre le sujet et l'objet direct de la phrase.

En effet, lors de l'évolution du latin vers les langues romanes, notamment après la disparition de la flexion latine et la neutralisation de l'opposition entre le nominatif et l'accusatif des noms, les langues romanes ont eu besoin de nouveaux moyens pour faire la distinction entre les éléments de la phrase, qui n'étaient plus marqués par des désinences flexionnelles (cf., entre autres, Drăganu (1943), Müller (1971), Sala (1999), Laca (2006) ${ }^{13}$ ).

Dans ce but, le français et l'italien ont adopté un ordre rigide des mots, tandis que le roumain et l'espagnol, gardant l'ordre relativement libre des mots qui caractérisait le latin, ont adopté un nouveau moyen pour distinguer entre le sujet et l'objet direct, à savoir les marques pe et $a$. Par conséquent, en roumain et en espagnol, pe et $a$ éviteraient l'ambiguïté possible entre le sujet et l'objet direct.

Il s'agit notamment des phrases où le sujet, le verbe et l'objet direct renvoient à la même personne grammaticale (c.-à-d. la troisième du singulier), ainsi que des phrases où le sujet et l'objet direct - qui sont formellement identiques peuvent tous les deux porter le rôle Agent. Les deux paradigmes suivants illustrent ces propos :

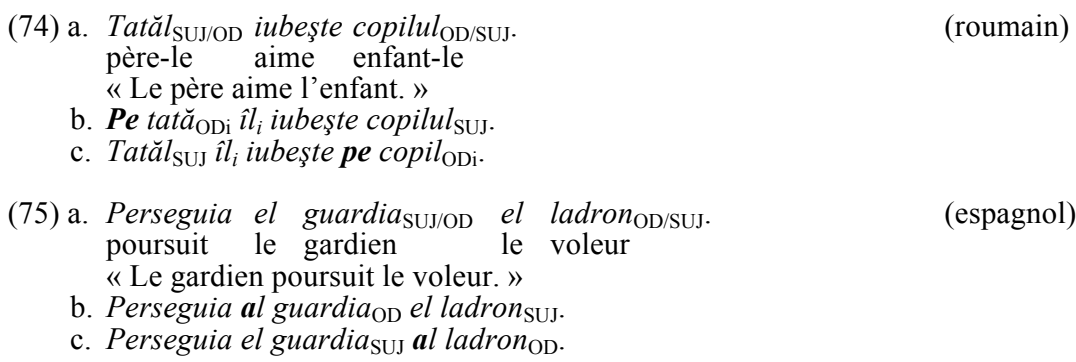

L'exemple roumain donné en (74a) est potentiellement ambigu, en ce sens que les groupes nominaux tatăl « le père » et copilul "l'enfant» peuvent être tous les deux analysés comme le sujet ou l'objet direct de la phrase. Il en est de même pour l'exemple espagnol donné en (75a), à cette différence près que les deux groupes nominaux sont tous les deux postverbaux.

L'insertion de pe et $a$ permet de marquer l'objet direct (comparons (74b) et (75b) avec (74c) et (75c)).

Cette analyse a l'avantage de pouvoir rendre compte non seulement des situations où l'objet direct réfère à des individus [+ humain], mais aussi de celles où le référent de l'objet direct est [- animé], comme dans les exemples suivants :

\footnotetext{
${ }^{13}$ Laca (2006) apud Müller (1971) note que la marque de l'objet direct apparaît déjà en latin vulgaire : uenerunt Gundessaluo et alio bassalo et prendiderunt ad Sancio et a Nunnu Gomiz « G. et les autres vassaux sont venus et ont amené S. et N. G. ».
} 


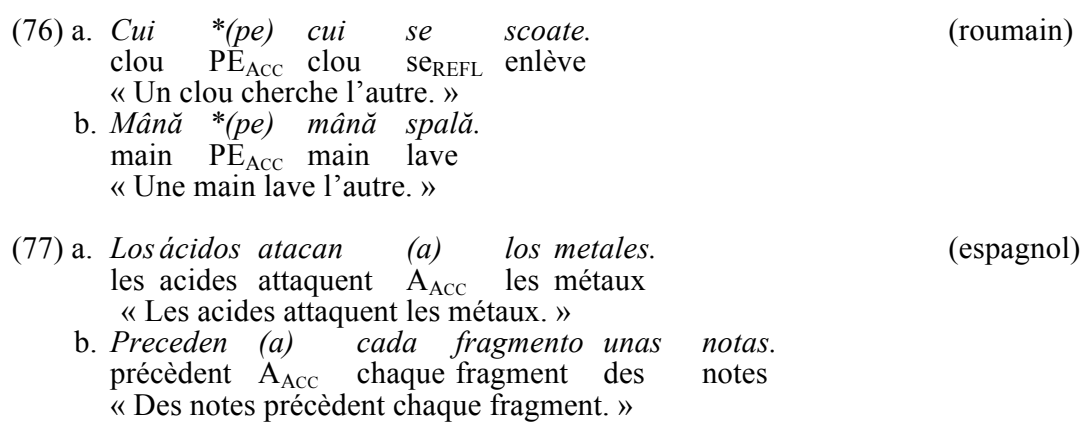

Toutefois, notons qu'il existe de nombreuses situations où il n'est pas question d'une telle ambiguité, malgré le caractère obligatoire de la marque. Il s'agit notamment des phrases à sujet nul ou encore des phrases où le sujet et l'objet direct ne renvoient pas à la même personne grammaticale (en l'occurrence, la première et la troisième du singulier) :

$$
\begin{aligned}
& \left(\hat{I} l_{i}\right) \quad v a ̆ d \quad *(p e) \quad I o n_{i} / \quad e l_{i} \text {. } \\
& \mathrm{le}_{\mathrm{CL} . \mathrm{ACC}} \text { vois } \mathrm{PE}_{\mathrm{ACC}} \text { Jean/ lui } \\
& \text { « Je vois Jean / je le vois. » } \\
& \text { (79) Veo *(a) Juan / él. }
\end{aligned}
$$

(espagnol, même traduction que (78))

\subsubsection{La thèse de l'analogie avec l'objet indirect ${ }^{14}$}

Les études qui avancent cette thèse notent qu'en espagnol la marque $a$ de l'objet direct se serait développée à partir de la même source que celle de l'objet indirect, c'est-à-dire la préposition $a$ 《 à ».

Meyer-Lübke (1899) montre que l'apparition de $a$ avec les objets directs a été initialement favorisée par la nature du verbe ${ }^{15}$. Plus précisément, ce sont les verbes à double régime - c'est-à-dire qui peuvent se combiner soit avec des objets indirects, soit avec des objets directs - qui ont joué un rôle important dans ce processus. Cette propriété des verbes à double régime a été également renforcée par l'existence des structures verbales coordonnées, dans lesquelles un objet précédé de $a$ peut être interprété tantôt comme direct, tantôt comme indirect, suivant le verbe dont il dépend. L'exemple suivant, tiré de Laca (2006), illustre ce phénomène :

\footnotetext{
${ }^{14}$ Parmi les auteurs qui soutiennent cette thèse, voir Cuervo (1886), Meyer-Lübke (1899), Hanssen (1913), Gili Gaya (1961), Lapesa (1964), Pensado (1985).

${ }^{15}$ Laca (2006) apud Müller (1971: 499) montre que ce phénomène était également présent en latin vulgaire : ecclesias [...] quod nos consecrare iussimus uel restaurare ad pontifice « l'église [...] que nous ordonnons de consacrer ou restituer au pape ».
} 
$(80)$

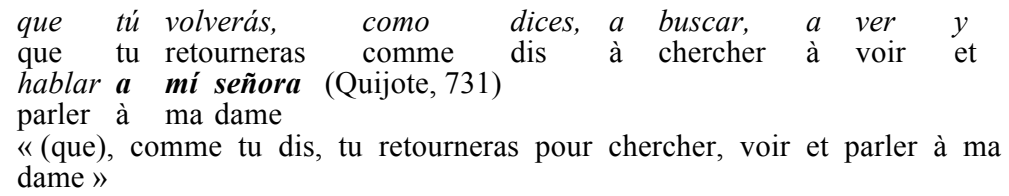

Lapesa (1964) compte, parmi les facteurs qui ont favorisé l'apparition de $a$ datif avec les objets accusatifs, la neutralisation de la distinction formelle entre le datif et l'accusatif des pronoms personnels atones, laquelle neutralisation s'est étendue par la suite aux formes toniques des pronoms.

Enfin, Pensado (1985) relie cette dernière observation au fait que les pronoms toniques de l'espagnol apparaissent toujours comme Topics - c'est-à-dire comme objets à référent connu, proéminent dans le discours -, d'où la nécessité d'être marqués.

\subsection{Les analyses sémantico-lexicales}

Les analyses que nous avons regroupées dans cette classe attribuent à $p e$ et $a$ le statut de marques dudit "genre personnel». Cette expression vient de la grammaire traditionnelle - elle a été proposée par Spitzer (1928) pour l'espagnol et développée par la suite par Racoviţă (1940) - et désigne les expressions nominales ayant le trait [+ humain $]^{16}$.

L'argument principal de ces analyses est fourni par l'étroite corrélation qui existe entre la présence de la marque et le trait [+ humain]. En effet, pe et $a$ apparaissent généralement avec des objets directs qui réfèrent à des entités humaines.

Comme nous l'avons déjà constaté dans les sections précédentes, cette corrélation n'est cependant pas systématique, puisqu'il existe, d'une part, des situations où la présence de la marque ne tient pas au trait mentionné, et, d'autre part, des situations où la présence du trait [+ humain] n'implique pas l'apparition de la marque.

Par exemple, certains pronoms du roumain qui sont obligatoirement précédés de pe ne réfèrent pas toujours à des humains. Dans (81) ci-dessous, le démonstratif acesta " ce » et le possessif al meu « le mien » peuvent référer à une entité non animée (p. ex., un camion) :

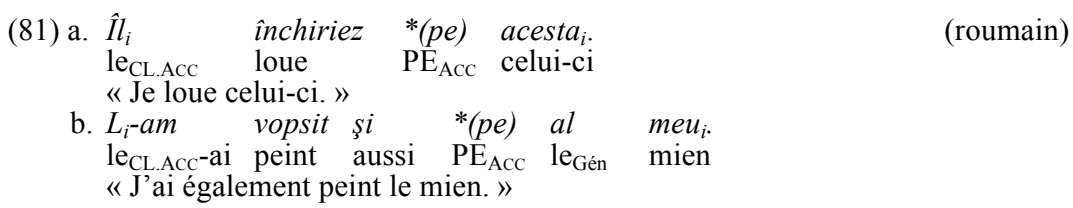

16 D'autres auteurs soutiennent cette thèse ; ce sont Hatcher (1942), Graur (1945), Niculescu (1959, 1965), GLR (1966), Pană Dindelegan (1997), Cornilescu (2000). 
Corrélativement, les noms propres ne réfèrent pas toujours à des humains. Dans ce sens, rappelons le cas du sarde, qui marque obligatoirement tous les objets directs réalisés comme noms propres, quelle que soit la nature de leur référent :

(82) Appo vistu *(a) Napoli.

$$
\begin{aligned}
& \text { ai vu } \mathrm{A}_{\mathrm{ACC}} \text { Naples } \\
& \text { "J'ai vu Naples. » }
\end{aligned}
$$

(sarde)

De même, il n'est pas vrai que tous les objets directs qui renvoient à des humains entraînent le marquage différentiel. Ainsi, les noms nus des trois langues mentionnées, quand ils apparaissent sans modifieurs, ne sont pas introduits par pe et par $a$, respectivement :

$$
\begin{array}{lll}
\text { Caut } & \left({ }^{*} p e\right) & \text { secretare. } \\
\text { cherche } & \mathrm{PE}_{\mathrm{ACC}} & \text { secrétaires }
\end{array}
$$
« Je cherche des secrétaires. »

(roumain)

(84) Detuvieron (*a) hinchas. arrêtèrent $\quad \mathrm{A}_{\mathrm{AcC}}$ supporters « Ils ont arrêté des supporters. »

(85) An assessinatu (*a) sordatos. ont assassiné A A Acc soldats
«Ils / elles ont assassiné des soldats. »

(sarde)

En outre, les objets directs réalisés comme expressions nominales définies référant à des humains n'entraînent pas toujours le marquage différentiel. Voici quelques exemples qui illustrent ce propos :

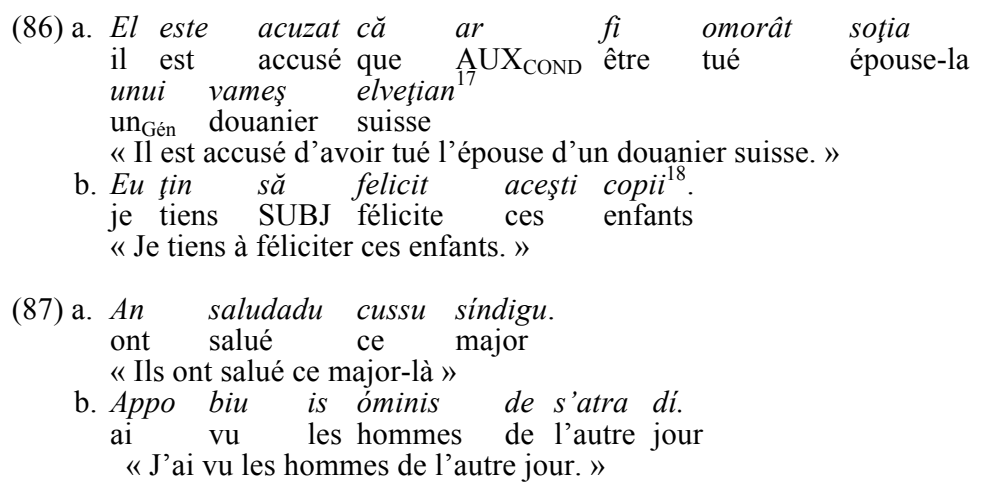

\footnotetext{
${ }_{18}^{17}$ Le Journal ProTV du 31 juillet 2003 à 8 h16.

${ }^{18}$ Realitatea TV, În direct cu olimpicii du $1^{\text {er }}$ août 2003 à $19 \mathrm{~h} 46$.
} 
Finalement, certains objets directs de l'espagnol peuvent être précédés de $a$, et ce malgré le caractère inanimé de leur référent. Voici un exemple tiré de Laca $(1995: 83))$ :

$$
\begin{aligned}
& \text { Una vez, a traversando el Pont Neuf, ví (a)l } \\
& \text { une fois en traversant le Pont Neuf vis } \mathrm{A}_{\mathrm{Acc}}-\mathrm{le} \\
& \text { barquito en cuestión. } \\
& \text { bateau en question } \\
& \text { "Un jour, pendant que je traversais le Pont-Neuf, j'ai vu le (petit) bateau en } \\
& \text { question. » }
\end{aligned}
$$

\subsection{Les analyses en termes de facteurs multiples}

La dernière classe d'analyses que nous présentons dans cette section est fondée sur l'idée que le marquage différentiel de l'objet ne peut pas être expliqué par un seul facteur. Comme nous l'avons observé dans la première partie de cette étude, l'existence de ce phénomène ne peut pas se ramener à une simple dichotomie entre deux contextes caractérisés soit par la présence soit par l'absence d'un trait quel qu'il soit. Autrement dit, il est clair que plusieurs facteurs qui favorisent le phénomène sont à l'œuvre simultanément.

De nombreux auteurs ${ }^{19}$ s'accordent sur le fait qu'il existe une hiérarchie de paramètres (ou facteurs) qui intervient dans le marquage différentiel de l'objet rappelons à ce point de notre discussion la distinction entre les paramètres globaux et locaux (section 2 ci-dessus). Cette hiérarchie permet de capter les propriétés des objets directs en vertu desquelles ces derniers doivent, peuvent ou ne peuvent pas être marqués.

Cette idée est clairement exprimée par Lazard (1994 : 230) :

(89) « la marque [...] se trouve toujours du côté du plus défini / plus humain et son absence du côté du moins défini / moins humain. Le marquage de l'objet est donc corrélatif de son individuation : plus l'objet est fortement individué, plus il a des chances d'être marqué comme tel »;

ou encore par Aissen (2003: 2) :

(90) « the higher in prominence a direct object, the more likely it is to be overtly case-marked ${ }^{20} »$.

Un autre aspect important concernant plus particulièrement le marquage différentiel de l'objet dans les langues romanes est que les paramètres qui influencent ce phénomène ne doivent pas être considérés individuellement. Afin d'aboutir à une description adéquate du phénomène, il faut croiser tous les

\footnotetext{
${ }^{19}$ Voir, entre autres, Bossong (1985, 1998), Croft (1988), Lazard (1994), Aissen (2003), von Heusinger \& Kaiser (2005), Laca (2006), von Heusinger \& Onea (2007).

${ }^{20}$ Plus le référent d'un objet direct est proéminent, plus ce dernier a des chances d'être casuellement marqué.
} 
paramètres mentionnés précédemment. Comme nous le verrons dans la section suivante, chacun de ces facteurs représente une condition nécessaire, mais non suffisante, pour qu'un objet direct soit marqué.

\section{VERS UNE GENERALISATION EN TERMES DE TYPES SEMANTIQUES}

Dans ce qui suit, nous présenterons une solution alternative pour exprimer les conditions dans lesquelles le marquage différentiel de l'objet a lieu dans les langues romanes.

Nous esquisserons une généralisation corrélant l'emploi de la marque avec le type sémantique des expressions nominales fonctionnant comme objets directs. Soulignons que ce type d'approche n'est pas nouveau, mais repose sur l'intuition de Niculescu $(1959,1965)$, qui a été récemment explicitée et formalisée par Laca (1995), Dobrovie-Sorin (1997, 2002), Bleam (2004, 2005), Dobrovie-Sorin \& Beyssade (2005), Cornilescu \& Dobrovie-Sorin (2007). Notons également que ces études portent sur le roumain et / ou l'espagnol, tandis que la généralisation que nous proposons concerne également le sarde.

Avant de procéder à cette entreprise, nous considérons qu'il est utile de passer en revue les analyses sémantiques des expressions nominales. Ainsi, il est unanimement accepté (voir, entre autres, Kamp (1981), Heim (1982), Kleiber \& al. (2001), Dobrovie-Sorin \& Beyssade (2004)) que les expressions nominales peuvent être analysées comme ayant trois types de dénotation :

(i) les expressions nominales dénotant des individus (type $<\mathrm{e}>)^{21}$,

(ii) les expressions nominales dénotant des propriétés (type $<$ e,t $>$ ),

(iii) les expressions nominales dénotant des quantifieurs généralisés (type $<<\mathrm{e}, \mathrm{t}>\mathrm{t}>)$.

Pour les besoins de notre présentation, nous adopterons cette classification sans rentrer dans les détails de l'analyse.

Revenant aux résultats des descriptions effectuées dans la section $\mathbf{2}$ ci-dessus, il serait à présent intéressant de corréler l'apparition de la marque pe ou $a$ avec les trois types sémantiques que nous venons de mentionner. Pour exprimer cette corrélation, la seule généralisation que l'on peut proposer est négative. Nous la formulons comme suit :

(91) En roumain, en espagnol (européen) et en sarde, les marques pe et a sont exclues avec les objets directs ayant une dénotation de type propriété (type $<\mathrm{e}, \mathrm{t}>$ ).

Cette généralisation permet de rendre compte de l'absence du marquage différentiel de l'objet dans un certain nombre de constructions dans les trois langues examinées. Il s'agit, plus précisément, des objets directs qui se réalisent comme noms nus non modifiés, indépendamment de la nature [+/- animé] de leur référent, ainsi que des groupes nominaux indéfinis non spécifiques. Autrement

${ }^{21}$ De l'anglais $e=$ entity (individu / entité), $t=$ truth value (valeur de vérité). 
dit, le marquage différentiel est exclu avec les objets directs qui ne donnent pas lieu à une présupposition d'existentialité. Ajoutons par ailleurs que les objets directs qui excluent le marquage différentiel sont susceptibles d'être sémantiquement incorporés (cf. Mardale (2007)) $)^{22}$.

La généralisation (91) permet également de faire des prédictions quant à la présence du marquage différentiel de l'objet. Ainsi, les objets directs qui ont une dénotation de type individu (type $<\mathrm{e}>$ ) ou quantifieur généralisé (type $<<\mathrm{e}, \mathrm{t}>\mathrm{t}>$ ) peuvent a priori entraîner le marquage.

Ainsi formulée, cette prédiction laisse entendre que tous les objets directs ayant cette propriété formelle sont susceptibles d'être marqués. Or, nous avons constaté que seuls les objets réalisés comme noms propres, en espagnol et en sarde, ou comme pronoms déictiques et anaphoriques, en roumain, entraînent le marquage différentiel, en vertu de leurs propriétés dénotationnelles.

En effet, le type dénotationnel de l'objet est une condition nécessaire, mais non suffisante, pour l'apparition du marquage différentiel. Comme nous l'avons constaté au cours de la description des données, il existe de nombreux objets directs de type individu ou quantifieur généralisé qui n'entraînent pas de marquage : c'est le cas des groupes nominaux définis, indéfinis ou encore des quantifieurs nus à référent non humain et / ou non animé. Dans le cas de ces derniers, la dénotation de l'objet direct n'entraîne pas à elle seule le marquage et doit, par conséquent, être corroborée par d'autres facteurs.

Il s'agit du caractère [+/- humain] et / ou [+/- animé] du référent de l'objet. C'est là une autre condition nécessaire pour l'apparition du marquage. Selon cette condition, les objets directs qui dénotent des individus ou des quantifieurs généralisés non humains ou non animés ne peuvent pas entraîner de marquage différentiel.

En revanche, les objets directs qui ont le type dénotationnel $<$ e $>$ et $<<e, t>$, $t>$ et qui renvoient à des référents [+ humain] ou [+ animé] peuvent et, dans certains cas, doivent recevoir le marquage. Ceci explique pourquoi les objets directs réalisés comme pronoms personnels, pronoms déictiques et anaphoriques à référent humain, groupes nominaux définis et indéfinis à référent humain ou encore comme quantifieurs nus à référent humain entraînent le marquage différentiel dans toutes les langues examinées.

Enfin, rappelons qu'à la seule exception de certains objets directs indéfinis de l'espagnol, l'apparition du marquage sur un objet direct donne lieu à une lecture de type spécifique, dans les trois langues en question.

\section{CONCLUSION}

Nous avons examiné, d'un point de vue comparatif, la distribution du marquage différentiel de l'objet dans les langues romanes. Nous avons concentré notre attention sur les trois langues qui présentent un usage étendu de ce phénomène, à savoir le roumain, l'espagnol (européen) et le sarde.

${ }^{22}$ Cf. également l'analyse en termes culioliens de Danon-Boileau \& Donabédian (1993). 
À la suite de Aissen (2003) et Laca (2002, 2006), nous avons constaté qu'il convient d'adopter une analyse en termes de hiérarchie des paramètres qui déterminent le phénomène. Nous avons également constaté qu'il convient d'établir une distinction entre les paramètres locaux (c.-à-d. ceux qui renvoient aux propriétés inhérentes de l'objet direct) et les paramètres globaux (c.-à-d. ceux qui renvoient aux propriétés du contexte dans lequel apparaît l'objet direct). Nous avons ainsi observé que le marquage différentiel de l'objet dans les langues romanes dépend des valeurs les plus hautes de la hiérarchie des paramètres locaux. Hormis ce comportement commun, nous avons aussi observé que le marquage différentiel de l'objet dans les trois langues étudiées peut être influencé par les valeurs de plusieurs paramètres globaux : certains objets qui ne sont pas a priori marqués en vertu de leurs propriétés inhérentes peuvent cependant recevoir le marquage différentiel lorsqu'ils sont soumis à l'influence d'un facteur global. Dans cette optique, nous avons montré que les langues en question se caractérisent par certains points de variation forte et de variation faible.

La variation forte concerne, pour la plupart du temps, le marquage des objets directs qui sont soumis à l'influence d'un facteur global. C'est notamment le cas de certains objets directs de l'espagnol dont le marquage peut être influencé par les propriétés lexicales du verbe et par l'interprétation du sujet. Comme nous l'avons montré, ces deux facteurs globaux n'influencent pas le marquage différentiel de l'objet en roumain et en sarde.

Le seul point de variation forte qui est dû à l'influence des facteurs locaux est le suivant : à la différence de l'espagnol et du sarde, le roumain n'est pas sensible au caractère $+/$ - animé de l'objet direct lorsque ce dernier est réalisé comme pronom déictique (ou anaphorique). Ainsi, l'espagnol et le sarde marquent uniquement les objets directs réalisés comme pronoms déictiques à référent humain, tandis que le roumain marque tous les objets directs réalisés comme pronoms déictiques ou anaphoriques, quelle que soit la nature de leur référent.

La variation faible concerne dans une égale mesure les facteurs locaux et les facteurs globaux. En ce qui concerne les premiers, il s'agit du marquage des objets directs occupant des positions situées vers le milieu de la hiérarchie, tels que les groupes nominaux définis et indéfinis. De ce point de vue, nous avons vu que l'espagnol a tendance à marquer plus facilement ces objets, lorsqu'ils ont un référent au moins animé. En revanche, le roumain et le sarde marquent de façon plus restreinte ce genre d'objet, en ce sens que ces deux langues sont sensibles, d'une part, au caractère humain de l'objet, et, d'autre part, au caractère défini et / ou spécifique. Pour ce qui est des facteurs globaux donnant lieu à une variation faible, nous avons constaté que les trois langues examinées marquent certains objets directs lorsqu'ils sont disloqués à gauche ou lorsqu'ils comportent des modifieurs. À nouveau, nous avons constaté que l'influence de ces facteurs est plus importante en espagnol (en ce sens que les types d'objet qui peuvent être marqués sont plus nombreux), tandis qu'elle est plus restreinte en roumain et encore moins en sarde. 
Finalement, après avoir passé en revue les analyses proposées pour rendre compte de ce phénomène, nous avons esquissé une hypothèse alternative, qui est basée sur le type sémantique de l'objet direct. Elle concerne exclusivement l'impossibilité d'apparition du marquage différentiel et peut être résumée comme suit : dans les langues romanes, le marquage différentiel de l'objet est exclu avec les objets qui ont une dénotation de type propriété (type $<\mathrm{e}, \mathrm{t}>$ ). Cette hypothèse permet de dériver les cas dans lesquels le marquage est possible, en l'occurrence pour les objets directs ayant une dénotation de type individu ou de quantifieur généralisé. Pour bon nombre de cas, ce dernier point n'est cependant pas une condition suffisante pour que le marquage différentiel se produise : comme nous l'avons constaté, il faut également prendre en compte le caractère [+/- humain] et [+/- animé] du référent de l'objet direct.

\section{BIBLIOGRAPHIE SELECTIVE}

Aissen J., 2003, Differential Object Marking: Iconicity vs. Economy, Natural Language and Linguistic Theory, 21, p. 435-483.

Beyssade C., 2007, Incorporation (sémantique), Sémanticlopédie, www.semantique-gdr.net/dico/index.php

Blasco Ferrer E., 1986, La lingua sarda contamporanea. Grammatica del logudorese e del campidanese. Norma e varietà dell'uso. Sintesi storica, Cagliari, Dell Torre.

Blasco Ferrer E. \& Ingrassia G., 2007, Sardo e lingue romanze a confronto. Nuove prospettive per la genesi dell'accusativo preposizionale, $X X V^{e}$ Congrès International de Linguistique et de Philologie Romanes, Innsbruck, les 3-8 septembre.

Bleam T., 2004, A Property Analysis of Weak Nominals in Spanish: Bare Nominals and Prepositionless Accusatives, manuscrit, Université Paris 7.

Bleam T., 2005, Two Cases of Unambiguously Property-denoting NPs in Spanish, Brussels Internatinal Conference: Indefinites and Weak Quantifiers, 6-8 January 2005.

Bosque Muñoz I. \& Demonte Barreto V. (coord.), 1999, Gramatica descriptiva de la lengua española, vol. 2, Espasa - Calpe, Madrid.

Bossong G., 1985, Empirische Universalienforschung. Differentielle Objektmarkierung in den neuiranischen Sprachen. Tübingen, Narr.

Bossong G., 1998, Le marquage différentiel de l'objet dans les langues d'Europe, in J. Feuillet (ed.), Actance et valence dans les langues de l'Europe. Berlin - New York, Mouton de Gruyter, 258-293.

Brugè L. \& Brugger G., 1994, On the Accusative A in Spanish, University of Venice WPL 4.1, p. 3-45.

Candrea I. A. \& Densusianu Ov., 1914, Dicţionarul etimologic al limbii române, Socec / Comp, București.

Comrie B., 1975, Definite and Animate Direct Objects: A Natural Class, Lingüística Silesiona 3, p. 13-21.

Cornilescu A., 2000, Notes on the Interpretation of the Prepositional Accusative in Romanian, Bucharest Working Papers in Linguistics, Departament of English, Faculty of Foreign Languages and Literatures, University of Bucharest, vol. II, nr. 1 / 2000, p. 91-10 
Cornilescu A. \& Dobrovie-Sorin C., 2007, Clitic Doubling. Complex Heads and Interarboreal Operation, manuscrit, Université de Bucarest / Paris 7.

Cuervo R., 1886, Diccionario de construccion y régimen de la lengua castellana, Paris.

Danon-Boileau L. \& Donabédian A., 1993, Construction référentielle et actance : l'exemple de l'arménien occidental, Bulletin de la Société Linguistique de Paris, t. 88, f. 1, p. 121-138.

Dayal V., 2003, A Semantics for Pseudo Incorporation, ms. Rutgers University.

Densusianu Ov., 1961, Istoria limbii române, vol. 1-2, Editura Ştiinţifică, București.

Diaconescu P., 1970, Acuzativul cu pre în textele traduse din secolul al XVI-lea in Structură şi evoluţie în morfologia substantivului românesc, Editura Academiei, București, p. 259-263.

Diez F., 1876, Grammaire des langues romanes, vol. III, A. Franck, Paris.

Dimitrescu F., 1960, Despre pre la acuzativ în limba textelor traduse din slavă în sec. al XVI-lea, Studii şi cercetări lingvistice, tomul XI, nr. 2, p. 219-226.

Dobrovie-Sorin C., 1987, Syntaxe du roumain. Chaînes thématiques, Thèse de Doctorat d'Etat, Université Paris 7.

Dobrovie-Sorin C., 1997, Classes de prédicats, distribution des indéfinis et la distinction thétique-catégorique, Le gré des langues 20, p. 58-97.

Dobrovie-Sorin C., 2002, From DPs to NPs: A Bare Phrase Account of Genitives, in M. Coene \& Y. d'Hulst (eds), From NP to DP. Volume 2, The expression of possession in noun phrases, Amsterdam / Philadelphia, John Benjamins.

Dobrovie-Sorin C. \& Laca B., 2003, Les noms sans déterminant dans les langues romanes, in Godard D. (éd.), Les langues romanes. Problèmes de la phrase simple, Editions du CNRS, p. 235-281.

Dobrovie-Sorin C. \& Beyssade C., 2004, Définir les indéfinis, Paris, Éditions du CNRS.

Dobrovie-Sorin C. \& Beyssade C., 2005, A syntax based analysis of predication, Journées de Sémantique et Modélisation 3, ENS Paris, les 17-18 mars.

Dobrovie-Sorin C., 2006, Number and Types of Semantic Incorporation, Proceedings of Nominal Incorporation and Its Kind, Ottawa.

Drăganu N., 1943, Morfemele româneşti ale complementului în acuzativ şi vechimea lor, lucrare postumă, Bucureşti.

Farkas D. \& de Swart H., 2003, The Semantics of Incorporation, CSLI Publications, Standford.

Farkas D. \& von Heusinger K., 2003, Stability of Reference and Object Marking in Romanian, ms., Universität Stuttgart.

Fish G., 1967, A with Spanish Direct Object, Hispania, 50, 1.

Floricic F., 2003, Notes sur l'accusatif prépositionnel, en Sarde, Bulletin de la Société de Linguistique de Paris, t. 98, fasc. I, p. 247-303.

GALR, 2005) = Gramatica Limbii Române, vol. I. Cuvântul, vol. II. Enunţul, Bucureşti, Editura Academiei Române.

van Geenhoven V., 1996, Semantic Incorporation and Indefinite Descriptions, PhD Thesis, Univ. Tubingen. Publiée en 1998, CSLI Publications, Standford.

Gili Gaya, 1961, Curso superior de sintaxis española, Barcelona.'

GLR, 1966) = Gramatica limbii române, vol. I-II, ediţia a doua, Bucureşti, Editura Academiei.

Gramatica Limbii Române, 1963, vol. 1-2, ediţia a 2-a revăzută şi adăugită, Editura Academiei RSR, Bucureşti. 
Graur A., 1945, Contributions à l'étude du genre personnel en roumain, Bulletin linguistique, nr. 13, p. 97-98.

Guțu Romalo V., 1973, Sintaxa limbii române. Probleme şi interpretări, Editura Didactică şi Pedagogică, Bucureşti.

Hanssen F., 1913, Gramatica historica de la lengua castellana, Halle, A. S.

Hatcher A., 1942, The Use of $a$ as a Designation of the Personal Accusative in Spanish, Modern Language Notes, 57, Baltimore, p. 421-429.

Heim I., 1982, The Semantics of Definite and Indefinite Descriptions, Doctoral Dissertation, University of Massachussets, Amherst (publiée en 1989 par Garland, New-York).

Hills E. C., 1920, The Accusative a, Hispania, 3, p. 216-222

von Heusinger K. \& Kaiser G. A., 2005, The evolution of differential object marking in Spanish, Proceedings of the Workshop: Specificity and the Evolution / Emergence of Nominal Determination Systems in Romance, Univ. Konstanz, p. 33-69.

von Heusinger K. \& Kaiser G. A., 2007, Differential object marking and the lexical semantics of verbs in Spanish, Workshop on DOM in Romance, Stuttgart, University of Stuttgart, les 14-15 juin.

von Heusinger K \& Onea E., 2007, The diachronic development of DOM in Romanian, Workshop on DOM in Romance, Stuttgart, University of Stuttgart, les 14-15 juin.

Iemmolo G., 2007, La marcatura differenziale dell'oggetto in siciliano: un'analisi contrastive, $X X V^{e}$ Congrès International de Linguistique et de Philologie Romanes, Innsbruck, les 3-8 septembre.

Ionescu E., 2000, The Role of pe in the Direct Object construction in Romanian (some critical remarks), Bucharest Working Papers in Linguistics, Departament of English, Faculty of Foreign Languages and Literatures, University of Bucharest, vol. II, nr. 1, p. 81-91.

Jaeggli O., 1986, Three Issues in the Theory of Clitics: Case, Doubled NPs, and Extraction, in Borer, H. (eds.), Syntax and semantics, 19, Academic Press, London, p. 15-42.

Jones M. A., 1993, Sardinian Syntax, London, Routledge.

Kamp H., 1981, A Theory of Truth and Discourse Representation, in J. Groenendijk, T. Janssen \& M. Stokhof (eds), Formal Methods in the Study of Language, Amsterdam, Mathematical Centre, p. 277-322.

Karimi S., 1990, Obliqueness, Specificity and Discourse Functions: $R \hat{a}$ in Persian, Linguistic Analysis 20.3-4, p. 139-191.

Karimi S., 1996, Case and Specificity. Persian Râ Revisited, Linguistic Analysis 26, p. 174-194

Laca B., 1995, Sobre el uso del acusativo preposicional en español, in Pensado, C., El complemento directo preposicional, Visor Libros, Madrid, p. 61-91.

Laca B., 2002, Gramaticalización y variabilidad - propriedades inherentes y factores contextuales en la evolución del acusativo preposiciónal en español, in A. Wesch (ed.), Sprachgeschichte als Varietätengeschichte romanicher Sprachen. Festschrift für Jens Lüdtke zum 60. Geburtstag, Tübingen, Stauffenburg, p. 195-303.

Laca B., 2006, El objeto directo, in C. Company (éd.), Sintaxis historica del español. Vol 1: La frase verbal, México, Universidad Nacional de México.

Landragin F., 2007, Saillance. Sémanticlopédie. Dictionnaire de sémantique, disponible à www.semantique-gdr.net/dico/index.php/Saillance

Lapesa R., 1959, Historia de la lengua española, Escélier, Madrid. 
Lazard G., 1982, Le morphème râ en persan et les relations actancielles, Bulletin de la Société de Linguistique de Paris, t. 77, fasc. I, p. 177-208.

Lenz R ., 1944, La oracion y sus partes, Madrid.

Leonetti M., 2003, Specificity and Differential Object Marking in Spanish, Catalan Journal of Linguistics 3, p. 75-114.

Leonetti M. (à paraître), Specificity in Clitic Doubling and in Differential Object Marking, Probvs.

Lois X., 1982, Sur l'Accusatif prépositionnel, mémoire de maîtrise, Université Paris 8.

Lujan M., 1977, Direct Object Nouns and the Preposition $a$ in Spanish, Texas Linguistic Forum, 10, 1978, p. 30-52.

Mardale A.-D., 2002, Analyse comparative de l'Accusatif prépositionnel: le roumain et l'espagnol, mémoire de maîtrise, Université Paris 7 / Universitatea din Bucureşti.

Mardale A., 2006, On the use of the definite article with prepositions in Romanian, Linguistic Symposium on Romance Languages 36, Rutgers University.

Mardale A., 2007, Microvariation within Differential Object Marking: Data from Romance, Linguistic Symposium on Romance Languages 37, University of Pittsburgh.

Massam D., 2001, Pseudo Noun Incorporation in Niuean, Natural Language and Linguistic Theory 19, p. 153-197.

McNally L., 1995, Bare Plurals in Spanish are Interpreted as Properties, in G. Morrill \&\& Oehrle D. (eds.), Proceedings of ESSLI Workshop on Formal Grammar, p. 197-222.

McNally L., 2004, Bare Plurals in Spanish are Interpreted as Properties, Catalan Journal of Linguistics 3, Universitat Politècnica de Catalunya, Barcelona, p. 115-133.

Meier H., 1947, O problema do accusativo preposicional no Catalão, Boletim de Filologia, 8 .

Meier H., 1948, Sobre as origines do accusativo preposicional nas linguas românicas, Ensaios de filologia românica, Lisboa.

Mensching G., 1994, Einführung in die sardische Sprache. Bonn, Romanistischer Verlag.

Mensching G., 2005, Remarks on Specificity and Related Categories in Sardinian, Proceedings of the Workshop: Specificity and the Evolution / Emergence of Nominal Determination Systems in Romance, Univ. Konstanz, p. 81-106.

Meyer-Lübke W., 1900, Grammaire des langues romanes, vol. 3, H. Welter, Paris.

Müller B., 1971, Das morphemmarkierte Satzobjekt der romanischen Sprachen. Der sogenannte präpositionale Akkusatif, Zeitschrift für romanische Philologie 87, p. 477-519.

Niculescu A., 1959, Sur l'objet direct prépositionnel dans les langues romanes, Recueil d'études romanes, $\mathrm{IX}^{\mathrm{e}}$ Congrès International de linguistique romane à Lisbonne, Bucarest, p. 103-124.

Niculescu A., 1965, Obiectul direct prepoziţional în limbile romanice, Individualitatea limbii române între limbile romanice, Editura Ştiinţifică, Bucureşti, p. 77-100. 
Onu L., 1959, L'origine de l'accusatif roumain avec $p(r)$ e, Recueil d'études romanes, IX ${ }^{\mathrm{e}}$ Congrès International de linguistique romane à Lisbonne, Bucarest, p. 188-209.

Pană Dindelegan G., 1997, Din nou despre statutul prepoziţiei. Cu referire specială la prepoziţia $P E$, Limba Română, XLVI, 1-3, ianuarie - iunie, p. $165-174$.

Pensado C., 1985, La creacion del Objeto directo preposicional y la flexion de los Pronombres Personales en las lenguas romanicas, Revue Roumaine de Linguistique, 30, Bucarest, p. 123-158.

Pensado C., 1995, El complemento directo preposicional, Visor Libros, Madrid.

Puşcariu S., 1922, Despre $p(r) e$ la acuzativ, Dacoromania, II, p. 565-581.

Racoviţă C., 1940, Sur le genre personnel en roumain, Bulletin linguistique, VII, p. 154 s.u.

Real Academia Española (RAE), 1973, Esbozo de una nueva gramatica de la lengua española, Espasa-Calpe, Madrid.

Roegiest E., 1979, Autour de l'accusatif prépositionnel dans les langues romanes, Vox Romanica 38, p. 37-54.

Rohlfs G., 1971, Autour de l'Áccusatif prépositionnel dans les langues romanes (concordances et discordances), RLiR, 35, p. 312-334

Rosetti A., 1973, Brève histoire de la langue roumaine des origines à nos jours, Mouton, The Hague, Paris.

Rosetti A., 1978, Istoria limbii române, Editura Stiintifică, Bucuresti.

Sala M. (coord.), 1989, Enciclopedia limbilor romanice, Bucureşti.

Spitzer L., 1928, Rum. P(r)e, Span. a vor persönalichem Akkusativobject, ArPh, 48, p. 423-432.

Torrego Salcedo E., 1999, El complemento directo preposicional, Bosque Muñoz I. \& Demonte Barreto V., Gramatica descriptiva de la lengua española, Espasa-Calpe, Madrid, p. 1779-1807. 